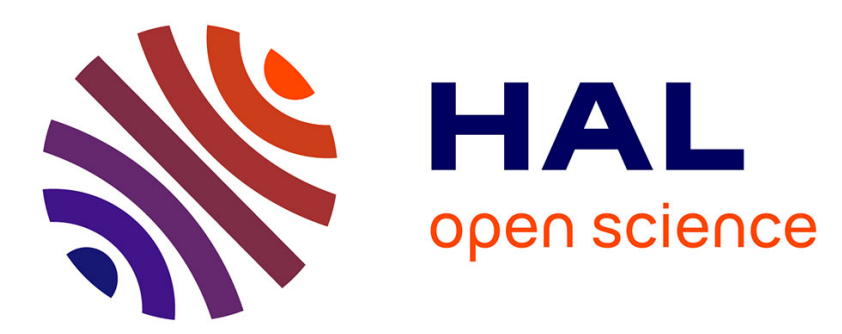

\title{
eXtended Stochastic Finite Element Method for the numerical simulation of heterogenous materials with random material interfaces
}

Anthony Nouy, Alexandre Clement

\section{- To cite this version:}

Anthony Nouy, Alexandre Clement. eXtended Stochastic Finite Element Method for the numerical simulation of heterogenous materials with random material interfaces. International Journal for Numerical Methods in Engineering, 2010, 83 (10), pp.1312-1344. 10.1002/nme.2865 . hal-00455646

\author{
HAL Id: hal-00455646 \\ https://hal.science/hal-00455646
}

Submitted on 11 Feb 2010

HAL is a multi-disciplinary open access archive for the deposit and dissemination of scientific research documents, whether they are published or not. The documents may come from teaching and research institutions in France or abroad, or from public or private research centers.
L'archive ouverte pluridisciplinaire HAL, est destinée au dépôt et à la diffusion de documents scientifiques de niveau recherche, publiés ou non, émanant des établissements d'enseignement et de recherche français ou étrangers, des laboratoires publics ou privés. 


\title{
eXtended Stochastic Finite Element Method for the numerical simulation of heterogenous materials with random material interfaces
}

\author{
A. Nouy*† and A. Clément \\ GeM - Research Institute in Civil Engineering and Mechanics, UMR CNRS 6183, \\ Centrale Nantes, University of Nantes, \\ 1 rue de la Nö̈, 44321 Nantes, France
}

\begin{abstract}
SUMMARY
An eXtended Stochastic Finite Element Method has been recently proposed for the numerical solution of partial differential equations defined on random domains. This method is based on a mariage between the eXtended Finite Element Method and spectral stochastic methods. In this paper, we propose an extension of this method for the numerical simulation of random multi-phased materials. The random geometry of material interfaces is described implicitly by using random level-set functions. A fixed deterministic finite element mesh, which is not conforming the random interfaces, is then introduced in order to approximate the geometry and the solution. Classical spectral stochastic finite element approximation spaces are not able to capture the irregularities of the solution field with respect to spatial and stochastic variables, which leads to a deterioration of the accuracy and convergence properties of the approximate solution. In order to recover optimal convergence properties of the approximation, we propose an extension of the partition of unity method to the spectral stochastic framework. This technique allows the enrichment of approximation spaces with suitable functions based on an a priori knowledge of the irregularities in the solution. Numerical examples illustrate the efficiency of the proposed method and demonstrate the relevance of the enrichment procedure.
\end{abstract}

KEY WORDS: Stochastic partial differential equations; Random geometry; Random Level-sets; XFEM; Spectral Stochastic Methods; Partition of Unity Method

\section{INTRODUCTION}

In the last two decades, a growing interest has been devoted to spectral stochastic methods $[1,2,3,4]$ for the propagation of uncertainties through physical models governed by stochastic partial differential equations (SPDEs) involving a finite dimensional noise. These methods are now relatively well mastered for the numerical solution of SPDEs with random operators or

\footnotetext{
*Correspondence to: Anthony Nouy : GeM, Centrale Nantes, 1 rue de la Noë, 44321 Nantes, France

${ }^{\dagger} \mathrm{E}-\mathrm{mail}$ : anthony.nouy@ec-nantes.fr

Grant sponsor: French National Research Agency; grant number: ANR-06-JCJC-0064
} 
right-hand sides $[5,6,7]$. However, only a few methods have been proposed for the propagation of geometrical uncertainties, which requires the solution of partial differential equations defined on random domains.

A first way to solve a PDE defined on a random domain consists in using direct spectral stochastic approaches (interpolation [8, 9, 10, 11, 12], projection [13, 14, 15], regression [16]), or any other direct stochastic method such as Monte-Carlo or quasi Monte-Carlo simulations [17], coupled with a deterministic finite element method with remeshings. This requires the numerical solution of a set of uncoupled deterministic PDEs, each solution requiring the generation of a new mesh conforming the geometry sample. These methods are quite easy to implement. However, they do not allow a complete description of the solution. Moreover, they may require to perform numerous deterministic computations, which leads to prohibitive computational costs.

Recently, spectral stochastic methods have been proposed in order to circumvent the above drawbacks. They are based on a reformulation of the problem on a deterministic reference domain. In [18], the authors introduce a random mapping between the random domain and a reference (non physical) domain. The initial PDE defined on a random domain is then transformed into a SPDE defined on a deterministic domain, where the random operator and right-hand side depend on the random mapping. Another method, based on the extension to the stochastic framework of the fictitious domain method, has been proposed in [19]. The main limitation of the above methods lies in the handling of complex geometries. In [20, 21], an eXtended Stochastic Finite Element Method (X-SFEM) has been proposed, which is based on the mariage between the deterministic X-FEM method [22, 23] and Galerkin spectral stochastic methods $[6,5]$. This method also starts with a reformulation of the problem on a fictitious deterministic domain and uses an implicit description of the random geometry with the levelset technique. This method allows the handling of complex geometries (eventually with changes in topology) and uses a fixed deterministic mesh for the definition of approximation spaces. Let us note that spectral stochastic methods provide an explicit description of the solution in terms of random parameters describing the geometrical uncertainties. In that sense, they can be seen as alternative methods for the construction of response surfaces, allowing different a posteriori analysis: sensitivity analysis with respect to shape parameters, shape optimization...

In this paper, we propose an extension of the X-SFEM method for the numerical solution of a PDE where the operator's parameters are discontinuous through a random interface. The random interface is described implicitly using the level-set technique. Then, the problem can be interpreted as a classical SPDE on a deterministic domain where the operator's parameters depend on a random level-set function. A fixed deterministic finite element mesh, which is not conforming the random interface, can then be introduced in order to approximate the geometry and the solution. However, classical spectral stochastic finite element approximation spaces (finite element approximation at the space level and generalized polynomial chaos at the stochastic level) are not able to capture the irregularities of the solution with respect to spatial and stochastic variables, which leads to very poor accuracy and convergence properties of the approximate solution. In the deterministic X-FEM framework, this drawback is circumvented by enriching the approximation spaces with the Partition of Unity Method (PUM) [24]. Approximation spaces are enriched with suitable additional functions whose selection is based on an a priori knowledge of the irregularities to be captured. In this article, we propose to extend the PUM to the spectral stochastic framework in order to define suitable approximation spaces which are able to capture the irregularities of the solution. This enrichment procedure is 
quite general and could lead to the developments of enriched spectral stochastic approximation techniques for other applications. Let us note that this kind of procedure has been used at the stochastic level in [25] for the solution of a random eigenvalue problem with spectral stochastic methods.

The remainder of the paper is organized as follows: in Section 2, we consider as a model problem a stationary diffusion equation for which the diffusion parameter is discontinuous through random interfaces separating phases of a material. In Section 3, we describe the implicit representation of the random geometry by the level-set technique. In Section 4, we briefly recall the basis of classical spectral stochastic methods and introduce two possible definitions of the approximate solution, respectively based on Galerkin and $L^{2}$ projections. In Section 5, we recall the basis of the partition of unity method for the construction of enriched approximation spaces and extend it to the spectral stochastic framework. Different enrichment strategies are introduced in Section 6 for the particular case of problems with random material interfaces. Finally, in Section 7, four numerical examples illustrate the accuracy of the approximate solutions obtained with different choices of approximation spaces and different projection techniques.

\section{FORMULATION OF THE PROBLEM}

\subsection{Strong formulation of the problem}

For clarity, the method is presented for a simple scalar stationary elliptic diffusion equation defined on a domain $\Omega \subset \mathbb{R}^{d}$, where the diffusion parameter is discontinuous through a random interface which separates two random subdomains, representing two phases of a material ${ }^{\dagger}$. This simple model problem highlights the essential features of the proposed methodology, which can be extended to a larger class of problems (e.g. elasticity problems in section 7 ). The extension of the method to more general elliptic boundary value problems, where the parameters of the differential operator are discontinuous through a random interface, is also straightforward. We suppose that the uncertainties on the geometry can be modeled with a finite set of random variables $\boldsymbol{\xi}$, with probability law $P_{\boldsymbol{\xi}}$. We introduce the associated probability space $\left(\boldsymbol{\Xi}, \mathcal{B}_{\boldsymbol{\Xi}}, P_{\boldsymbol{\xi}}\right)$, where $\boldsymbol{\Xi} \subset \mathbb{R}^{m}$ is the set of elementary events and $\mathcal{B}_{\boldsymbol{\Xi}}$ is a $\sigma$-algebra on $\boldsymbol{\Xi}$. The strong formulation of the problem writes: find the solution field $u$ such that it verifies almost surely

$$
\begin{aligned}
& -\nabla \cdot(\kappa \nabla u)=f \quad \text { on } \quad \Omega \\
& \kappa \nabla u \cdot n=g \quad \text { on } \quad \Gamma_{2} \\
& u=0 \quad \text { on } \Gamma_{1}
\end{aligned}
$$

where $\kappa$ is the diffusion parameter, $f$ is a body source, $g$ is a normal flux imposed on a part $\Gamma_{2}$ of the boundary $\partial \Omega$ ( $n$ is the unit outward normal to the boundary). We consider that the domain $\Omega$ is the union of two random subdomains $\Omega^{1}(\boldsymbol{\xi})$ and $\Omega^{2}(\boldsymbol{\xi})$, where the $\Omega^{k}: \boldsymbol{\xi} \in \boldsymbol{\Xi} \mapsto \Omega^{k}(\boldsymbol{\xi}) \subset \mathbb{R}^{d}$ are random variables modeling the geometrical uncertainties. We denote by $\Gamma(\boldsymbol{\xi})=\partial \Omega^{1}(\boldsymbol{\xi}) \cap \partial \Omega^{2}(\boldsymbol{\xi})$ the random interface which separates the two phases of the material. We consider that the diffusion parameter is discontinuous through $\Gamma(\boldsymbol{\xi})$ and is such

${ }^{\dagger}$ The extension of the formulation and of the proposed method to the case of multiple phases is straightforward. 
that

$$
\kappa(\boldsymbol{x}, \boldsymbol{\xi})=\left\{\begin{array}{l}
\kappa_{1} \text { if } \boldsymbol{x} \in \Omega^{1}(\boldsymbol{\xi}) \\
\kappa_{2} \text { if } \boldsymbol{x} \in \Omega^{2}(\boldsymbol{\xi})
\end{array}\right.
$$

where $\kappa_{1}$ and $\kappa_{2}$ are diffusion parameters associated with subdomains $\Omega^{1}$ and $\Omega^{2}$ respectively. Let us note that uncertainties on material parameters and sources may be classically introduced by considering $f, g, \kappa_{1}$ and $\kappa_{2}$ as random quantities (random variables or fields). For simplicity, we will consider in this paper that these quantities are deterministic and that the only source of uncertainty is the geometrical uncertainty.

\subsection{Weak formulation of the problem}

Let us introduce the deterministic function space $\mathcal{V}=\left\{v \in H^{1}(\Omega) ; v_{\mid \Gamma_{1}}=0\right\}$. The random solution $u$ is then searched in function space

$$
\mathcal{W}=L^{2}\left(\boldsymbol{\Xi}, d P_{\boldsymbol{\xi}} ; \mathcal{V}\right):=\left\{u: \boldsymbol{\xi} \in \boldsymbol{\Xi} \mapsto u(\cdot, \boldsymbol{\xi}) \in \mathcal{V} ; E\left(\|u(\cdot, \boldsymbol{\xi})\|_{\mathcal{V}}^{2}\right)<\infty\right\},
$$

where $E$ denotes the mathematical expectation, defined by $E(f(\boldsymbol{\xi}))=\int_{\Xi} f(\mathbf{y}) d P_{\boldsymbol{\xi}}(\mathbf{y})$. Function space $\mathcal{W}$ is isomorphic to the following tensor product space

$$
\mathcal{W} \simeq \mathcal{V} \otimes \mathcal{S}, \quad \mathcal{S}=L^{2}\left(\boldsymbol{\Xi}, d P_{\boldsymbol{\xi}}\right),
$$

where $\mathcal{S}$ is the space of second-order real-valued random variables defined on $\left(\boldsymbol{\Xi}, \mathcal{B}_{\boldsymbol{\Xi}}, P_{\boldsymbol{\xi}}\right)$. The weak formulation of (1) writes:

$$
u \in \mathcal{W}, \quad A(u, v)=B(v) \quad \forall v \in \mathcal{W}
$$

where

$$
\begin{aligned}
& A(u, v)=\sum_{k=1}^{2} \int_{\Xi} \int_{\Omega^{k}(\mathbf{y})} \nabla v \cdot \kappa_{k} \cdot \nabla u d x d P_{\boldsymbol{\xi}}(\mathbf{y}) \\
& B(v)=\int_{\Xi} \int_{\Omega} v f d x d P_{\boldsymbol{\xi}}+\int_{\Xi} \int_{\Gamma_{2}} v g d s d P_{\boldsymbol{\xi}}(\mathbf{y})
\end{aligned}
$$

Under classical regularity assumptions on sources $f, g$ and on diffusion parameters $\kappa_{i}$, bilinear form $A$ is continuous and coercive on $\mathcal{W}$ and linear form $B$ is continuous on $\mathcal{W}$, such that problem (4) is well posed (see e.g. [5]).

\section{MODELING RANDOM INTERFACES WITH THE LEVEL-SET TECHNIQUE}

In this section, we present a first important ingredient of the X-SFEM method: the implicit representation of the random geometry with the level-set technique [26].

\subsection{Random level-sets}

The geometry is implicitly represented by using the level-set technique. It consists in representing a hyper-surface contained in the domain $\Omega$ by the iso-zero of a function $\phi$, called 
a level-set function. A random hyper-surface $\Gamma(\boldsymbol{\xi})$ is here represented by a random level-set $\phi(\boldsymbol{x}, \boldsymbol{\xi}): \Omega \times \boldsymbol{\Xi} \rightarrow \mathbb{R}$ such that

$$
\Gamma(\boldsymbol{\xi})=\{\boldsymbol{x} \in \Omega ; \phi(\boldsymbol{x}, \boldsymbol{\xi})=0\} .
$$

By convention, we consider that the level-set function $\phi(\cdot, \boldsymbol{\xi})$ takes negative values in $\Omega^{1}(\boldsymbol{\xi})$ and positive values in $\Omega^{2}(\boldsymbol{\xi})$. Then, the two random subdomains $\Omega^{1}(\boldsymbol{\xi})$ and $\Omega^{2}(\boldsymbol{\xi})$ can be characterized by:

$$
\Omega^{1}(\boldsymbol{\xi})=\{\boldsymbol{x} \in \Omega ; \phi(\boldsymbol{x}, \boldsymbol{\xi})<0\}, \quad \Omega^{2}(\boldsymbol{\xi})=\{\boldsymbol{x} \in \Omega ; \phi(\boldsymbol{x}, \boldsymbol{\xi})>0\}
$$

Bilinear form $A$ in problem (4) can then be rewritten:

$$
A(u, v)=\int_{\Xi} \int_{\Omega} \nabla v \cdot\left(\kappa_{1} \mathcal{H}(-\phi(\boldsymbol{x}, \mathbf{y}))+\kappa_{2} \mathcal{H}(\phi(\boldsymbol{x}, \mathbf{y}))\right) \cdot \nabla u d x d P_{\boldsymbol{\xi}}(\mathbf{y})
$$

where $\mathcal{H}: \mathbb{R} \rightarrow\{0,1\}$ is the Heaviside function defined by:

$$
\mathcal{H}(y)= \begin{cases}1 & \text { if } y>0 \\ 0 & \text { if } y \leqslant 0\end{cases}
$$

Let us note that this level-set representation allows to handle complex topologies of phases (non-simply connected phases).

\subsection{Discretization of level-sets}

In practise, the level-set is discretized at the space level by introducing a finite element mesh $\mathcal{T}_{h}$ of the domain $\Omega$. We denote by $\left\{\varphi_{i}\right\}_{i \in I}$ the set of finite element interpolation functions associated with $\mathcal{T}_{h}$. The discretized level-set writes

$$
\phi(\boldsymbol{x}, \boldsymbol{\xi})=\sum_{i \in I} \varphi_{i}(\boldsymbol{x}) \phi_{i}(\boldsymbol{\xi})
$$

where the $\phi_{i}(\boldsymbol{\xi})$ are the nodal values of the random level-set, which are random variables. In this article, we consider linear finite element interpolation functions, thus resulting in a piecewise linear approximation of the interface.

Remark 1. In this article, we consider that the probabilistic model of the geometry is given. Let us note that in [27], a method has been introduced for the identification of a random geometry from sample images. It is based on the identification of a polynomial chaos expansion of the random level-set function $\phi$ whose samples are obtained from image recovery techniques. This identified random level-set could then be considered as the input of the method proposed in this article.

\section{CLASSICAL STOCHASTIC FINITE ELEMENT APPROXIMATION}

\subsection{Approximation spaces}

An approximation space $\mathcal{W}_{h, P} \subset \mathcal{W}=\mathcal{V} \otimes \mathcal{S}$ is classically obtained by tensorization of approximation spaces $\mathcal{V}_{h} \subset \mathcal{V}$ and $\mathcal{S}_{P} \subset \mathcal{S}$, i.e.

$$
\mathcal{W}_{h, P}=\mathcal{V}_{h} \otimes \mathcal{S}_{P}
$$


Here, we choose for $\mathcal{V}_{h}$ a finite element approximation space associated with a mesh $\mathcal{T}_{h}$ of $\Omega$ :

$$
\mathcal{V}_{h}=\left\{v(\boldsymbol{x})=\sum_{i=1}^{N} \varphi_{i}(\boldsymbol{x}) v_{i}, v_{i} \in \mathbb{R}\right\},
$$

where the $\varphi_{i}(\boldsymbol{x}) \in \mathcal{V}$ are the finite element basis functions. For $\mathcal{S}_{P}$, classical choices consists of polynomial spaces [28, 29, 9], piecewise polynomial spaces [30,31,32] or more general orthogonal bases [33]. Let $\left\{H_{\alpha}\right\}_{\alpha=1}^{P}$ denote a basis of $\mathcal{S}_{P}$ :

$$
\mathcal{S}_{P}=\left\{v(\boldsymbol{\xi})=\sum_{\alpha=1}^{P} v_{\alpha} H_{\alpha}(\boldsymbol{\xi}), \quad v_{\alpha} \in \mathbb{R}\right\}
$$

Finally, the tensor product approximation space $\mathcal{W}_{h, P} \subset \mathcal{W}$ is simply defined by:

$$
\begin{aligned}
\mathcal{W}_{h, P} & =\left\{v(\boldsymbol{x}, \boldsymbol{\xi})=\sum_{i=1}^{N} \varphi_{i}(\boldsymbol{x}) v_{i}(\boldsymbol{\xi}), v_{i} \in \mathcal{S}_{P}\right\} \\
& =\left\{v(\boldsymbol{x}, \boldsymbol{\xi})=\sum_{i=1}^{N} \sum_{\alpha=1}^{P} \varphi_{i}(\boldsymbol{x}) H_{\alpha}(\boldsymbol{\xi}) v_{i, \alpha}, v_{i, \alpha} \in \mathbb{R}\right\}
\end{aligned}
$$

\subsection{Galerkin approximation (X-SFEM-G)}

4.2.1. Definition The Galerkin approximation $u_{h, P} \in \mathcal{W}_{h, P}$ of problem (4) is defined by

$$
A\left(u_{h, P}, v_{h, P}\right)=B\left(v_{h, P}\right) \quad \forall v_{h, P} \in \mathcal{W}_{h, P}
$$

A function $u \in \mathcal{W}_{h, P}$ is assimilated with a random vector $\mathbf{u}: \boldsymbol{\Xi} \rightarrow \mathbb{R}^{N}$, such that $u(\boldsymbol{x}, \boldsymbol{\xi})=$ $\sum_{i=1}^{N} \varphi_{i}(\boldsymbol{x})(\mathbf{u}(\boldsymbol{\xi}))_{i}$. Equation (15) can then be reformulated as follows: find $\mathbf{u} \in \mathbb{R}^{N} \otimes \mathcal{S}_{P}$ such that

$$
E\left(\mathbf{v}^{T} \mathbf{A} \mathbf{u}\right)=E\left(\mathbf{v}^{T} \mathbf{b}\right) \quad \forall \mathbf{v} \in \mathbb{R}^{N} \otimes \mathcal{S}_{P},
$$

where the components of random matrix $\mathbf{A}$ and vector $\mathbf{b}$ are defined by

$$
\begin{aligned}
& (\mathbf{A}(\boldsymbol{\xi}))_{i j}=\int_{\Omega} \nabla \varphi_{j} \cdot\left(\kappa_{1} \mathcal{H}(-\phi(\boldsymbol{x}, \boldsymbol{\xi}))+\kappa_{2} \mathcal{H}(\phi(\boldsymbol{x}, \boldsymbol{\xi}))\right) \cdot \nabla \varphi_{i} d x \\
& (\mathbf{b}(\boldsymbol{\xi}))_{i}=\int_{\Omega} \varphi_{i} f d x+\int_{\Gamma_{2}} \varphi_{i} g d s
\end{aligned}
$$

4.2.2. Computational aspects for the Galerkin projection The solution $\mathbf{u}$ of (16) is written $\mathbf{u}(\boldsymbol{\xi})=\sum_{\alpha=1}^{P} \mathbf{u}_{\alpha} H_{\alpha}(\boldsymbol{\xi})$, where the set of coefficients $\mathbf{u}_{\alpha}$ is solution of the following system of $P \times N$ equations:

$$
\sum_{\alpha=1}^{P} E\left(\mathbf{A} H_{\alpha} H_{\beta}\right) \mathbf{u}_{\alpha}=E\left(\mathbf{b} H_{\beta}\right) \quad \forall \beta \in\{1 \ldots P\} .
$$

In practise, matrices $E\left(\mathbf{A} H_{\alpha} H_{\beta}\right)$ and vectors $E\left(\mathbf{b} H_{\beta}\right)$ are obtained by assembling elementary quantities $E\left(\mathbf{A}_{K} H_{\alpha} H_{\beta}\right)$ and $E\left(\mathbf{b}_{K} H_{\beta}\right)$ associated with finite elements $K \subset \mathcal{T}_{h}$. When 
considering deterministic sources $f$ and $g$, element vector $\mathbf{b}_{K}$ is deterministic. An approximation of $E\left(\mathbf{A}_{K} H_{\alpha} H_{\beta}\right)$ is obtained by introducing a quadrature rule $\left(\omega_{q}, \mathbf{y}_{q}\right)_{q=1}^{Q}$ on $\left(\boldsymbol{\Xi}, \mathcal{B}_{\boldsymbol{\Xi}}, P_{\boldsymbol{\xi}}\right)$ :

$$
E\left(\mathbf{A}_{K} H_{\alpha} H_{\beta}\right) \approx \sum_{q=1}^{Q} \omega_{q} \mathbf{A}_{K}\left(\mathbf{y}_{q}\right) H_{\alpha}\left(\mathbf{y}_{q}\right) H_{\beta}\left(\mathbf{y}_{q}\right)
$$

where $\mathbf{A}_{K}\left(\mathbf{y}_{q}\right)$ corresponds to the element matrix associated with a particular outcome $\boldsymbol{\xi}=\mathbf{y}_{q}$. For a given outcome, element matrix $\mathbf{A}_{K}\left(\mathbf{y}_{q}\right)$ is easily computed by using classical spatial integration (Gaussian quadrature on $K$ or on a partition of $K$ if the element is cut by the interface). However, a particular care must be taken for the choice of the stochastic quadrature rule. Indeed, element matrix $\mathbf{A}_{K}(\boldsymbol{\xi})$ appears to be a non-smooth function of $\boldsymbol{\xi}$, defined by

$$
\left(\mathbf{A}_{K}(\boldsymbol{\xi})\right)_{i j}=\left\{\begin{array}{lr}
\int_{K} \nabla \varphi_{j} \cdot \kappa_{1} \cdot \nabla \varphi_{i} & \text { for } \boldsymbol{\xi} \in \mathbf{\Xi}_{1}^{K} \\
\int_{K} \nabla \varphi_{j} \cdot \kappa_{2} \cdot \nabla \varphi_{i} & \text { for } \boldsymbol{\xi} \in \mathbf{\Xi}_{2}^{K} \\
\int_{K} \nabla \varphi_{j} \cdot\left(\kappa_{1} \mathcal{H}(-\phi(\boldsymbol{x}, \boldsymbol{\xi}))+\kappa_{2} \mathcal{H}(\phi(\boldsymbol{x}, \boldsymbol{\xi}))\right) \cdot \nabla \varphi_{i} d x & \text { for } \quad \boldsymbol{\xi} \in \mathbf{\Xi}_{c}^{K}
\end{array}\right.
$$

where $\boldsymbol{\Xi}_{1}^{K}=\{\boldsymbol{\xi} \in \boldsymbol{\Xi} ; \forall \boldsymbol{x} \in K, \phi(\boldsymbol{x}, \boldsymbol{\xi})<0\}$ is the set of elementary events $\boldsymbol{\xi}$ such that $K \subset \Omega^{1}(\boldsymbol{\xi}), \boldsymbol{\Xi}_{2}^{K}=\{\boldsymbol{\xi} \in \boldsymbol{\Xi} ; \forall \boldsymbol{x} \in K, \phi(\boldsymbol{x}, \boldsymbol{\xi})>0\}$ is the set of elementary events $\boldsymbol{\xi}$ such that $K \subset \Omega^{2}(\boldsymbol{\xi})$ and $\boldsymbol{\Xi}_{c}^{K}=\{\boldsymbol{\xi} \in \boldsymbol{\Xi} ; \exists \boldsymbol{x} \in K ; \phi(\boldsymbol{x}, \boldsymbol{\xi})=0\}$ is the set of elementary events $\boldsymbol{\xi}$ for which the element $K$ is cut by the interface $\Gamma(\boldsymbol{\xi})$. $\mathbf{A}_{K}(\boldsymbol{\xi})$ is constant on $\boldsymbol{\Xi}_{1}^{K}$ and $\boldsymbol{\Xi}_{2}^{K}$, and is a smooth function of $\boldsymbol{\xi}$ on $\boldsymbol{\Xi}_{c}^{K}$ (more precisely, the smoothness of $\mathbf{A}_{K}(\boldsymbol{\xi})$ depends on the smoothness of $\phi$ with respect to $\boldsymbol{\xi})$. In order to accurately compute $E\left(\mathbf{A}_{K} H_{\alpha} H_{\beta}\right)$, a suitable integration technique has been proposed in [21], which consists in introducing a Gaussian quadrature defined on a partition of $\boldsymbol{\Xi}$ which approximates the partition $\left\{\boldsymbol{\Xi}_{1}^{K}, \boldsymbol{\Xi}_{2}^{K}, \boldsymbol{\Xi}_{c}^{K}\right\}$. Let us note that the calculation of $E\left(\mathbf{A}_{K} H_{\alpha} H_{\beta}\right)$ can be performed independently on each finite element $K$. Therefore, this calculation step can be completely parallelized.

Finally, system (16) is a system of $P \times N$ which can be solved by using classical Krylov-type iterative solvers $[1,34]$ or alternative solution techniques such as the Generalized Spectral Decomposition method [35, 36, 37]. In this article, we do not focus on these numerical aspects and consider that an accurate solution of (16) is obtained with a traditional Krylov-type solver (preconditioned conjugate gradient).

\section{3. $L^{2}$ projection $(X-S F E M-P)$}

An alternative definition of the approximation consists in using a $L^{2}$ projection method at the stochastic level $[13,14,15]$. The approximation $u_{h, P} \in \mathcal{W}_{h, P}$ is defined by the $L^{2}$ projection of the semi-discretized solution $\mathbf{u} \in \mathbb{R}^{N} \otimes \mathcal{S}$ onto the subspace $\mathbb{R}^{N} \otimes \mathcal{S}_{P}$. The projection is defined with respect to the usual inner product on $\mathbb{R}^{N} \otimes L^{2}\left(\boldsymbol{\Xi}, d P_{\boldsymbol{\xi}}\right)$ :

$$
<\mathbf{u}, \mathbf{v}>_{L^{2}}=E\left(\mathbf{u}(\boldsymbol{\xi})^{T} \mathbf{v}(\boldsymbol{\xi})\right)=\int_{\boldsymbol{\Xi}} \mathbf{u}(\mathbf{y})^{T} \mathbf{v}(\mathbf{y}) d P_{\boldsymbol{\xi}}(\mathbf{y})
$$

When considering an orthonormal basis $\left\{H_{\alpha}\right\}_{\alpha=1}^{P}$ of $\mathcal{S}_{P}$, the coefficients $\mathbf{u}_{\alpha} \in \mathbb{R}^{N}$ of the approximate solution $\mathbf{u}(\boldsymbol{\xi})=\sum_{\alpha=1}^{P} \mathbf{u}_{\alpha} H_{\alpha}(\boldsymbol{\xi})$ are then defined by

$$
\mathbf{u}_{\alpha}=<\mathbf{u}, H_{\alpha}>_{L^{2}}=E\left(\mathbf{u}(\boldsymbol{\xi}) H_{\alpha}(\boldsymbol{\xi})\right)
$$


which are approximated by using a quadrature rule $\left(\omega_{q}, \mathbf{y}_{q}\right)_{q=1}^{Q}$ on $\left(\boldsymbol{\Xi}, \mathcal{B}_{\Xi}, P_{\boldsymbol{\xi}}\right)$ :

$$
\mathbf{u}_{\alpha} \approx \sum_{q=1}^{Q} \omega_{q} \mathbf{u}\left(\mathbf{y}_{q}\right) H_{\alpha}\left(\mathbf{y}_{q}\right)
$$

where the $\mathbf{u}\left(\mathbf{y}_{q}\right)$ are the solutions of $Q$ independent deterministic equations:

$$
\mathbf{A}\left(\mathbf{y}_{q}\right) \mathbf{u}\left(\mathbf{y}_{q}\right)=\mathbf{b}\left(\mathbf{y}_{q}\right)
$$

Remark 2. Let us note that in the case of problems with random shapes, this $L^{2}$ projection method leads to a very poor accuracy. This has been illustrated and explained in [21].

\subsection{Limitations of classical approximation spaces}

In a deterministic framework, it is well known that the accuracy and convergence properties of a finite element approximation deteriorate if the mesh is not conforming the material interface. Indeed, the gradient of the solution field is discontinuous through the interface. This discontinuity is naturally captured by a finite element approximation associated with a mesh conforming the interface. However, it is not captured if the material interface crosses the finite elements. Moreover, this lack of regularity in the solution is also observed at the stochastic level, where function $\boldsymbol{\xi} \mapsto u(\boldsymbol{x}, \boldsymbol{\xi})$ appears as a non-smooth function of $\boldsymbol{\xi}$ for points $\boldsymbol{x}$ such that $P_{\boldsymbol{\xi}}\left(\boldsymbol{x} \in \Omega^{1}(\boldsymbol{\xi})\right)>0$ and $P_{\boldsymbol{\xi}}\left(\boldsymbol{x} \in \Omega^{2}(\boldsymbol{\xi})\right)>0$. This also leads to a deterioration of accuracy and convergence properties of the approximation when using classical spectral approximations (e.g. polynomial chaos) at the stochastic level.

In the deterministic eXtended Finite Element Method, this drawback is circumvented by enriching the approximation space with suitable functions which capture the discontinuities in the solution [23,38]. This enrichment procedure is based on the partition of unity method [24]. In the following section, we propose to extend this methodology to the stochastic framework, in order to capture irregularities in the solution with respect to both spatial variable $\boldsymbol{x}$ and stochastic variable $\boldsymbol{\xi}$.

\section{THE PARTITION OF UNITY METHOD FOR THE CONSTRUCTION OF APPROXIMATION SPACES}

The partition of unity method (PUM) [24] provides a general framework for the construction of approximation spaces. In this section, we introduce a natural extension of the PUM for the construction of approximation spaces in tensor product space $\mathcal{W}=\mathcal{V} \otimes \mathcal{S}$. It provides a general methodology for the enrichment of classical stochastic finite element approximation spaces. Specific enrichment procedures will be introduced in the Section 6 for the case of random material interfaces.

\subsection{Construction of approximation space in $H^{1}(\Omega)$}

We first recall the construction of an approximation space of $H^{1}(\Omega)$ based on the partition of unity method. The first point consists in introducing a cover $\left\{\Omega_{i}\right\}_{i \in I}$ of $\Omega$. We then introduce a set of functions $\left\{\varphi_{i}\right\}_{i \in I}$ defined on $\Omega$ and verifying the following properties (partition of 
unity):

$$
\operatorname{supp}\left(\varphi_{i}\right) \subset \Omega_{i}, \quad \sum_{i \in I} \varphi_{i}=1 \quad \text { on } \quad \Omega
$$

An approximation space $V$ of $H^{1}(\Omega)$ can then be defined by

$$
V=\sum_{i \in I} \varphi_{i} V_{i}
$$

where the $V_{i} \subset H^{1}\left(\Omega_{i} \cap \Omega\right)$ are function spaces to be defined. The linear finite element interpolation functions $\left\{\varphi_{i}\right\}_{i \in I}$ associated with the nodes $\left\{\boldsymbol{x}_{i}\right\}_{i \in I}$ of a mesh $\mathcal{T}_{h}$ of $\Omega$ form a partition of unity associated with the cover $\left\{\Omega_{i}\right\}_{i \in I}$ composed by the supports of functions $\varphi_{i} . \Omega_{i}$ is composed by the union of elements $K \in \mathcal{T}_{h}$ containing the node $\boldsymbol{x}_{i}$ of the mesh. If one chooses for $V_{i}$ the space of constant functions, the space $V$ coincides with the space spanned by the interpolation functions $\varphi_{i}$, i.e. the classical finite element space. If one chooses for $V_{i}$ the space $\mathbb{Q}_{q}\left(\Omega_{i}\right)$ of polynomial functions of degree $q$, the space $V$ is a (spectral) finite element space with degree $q+1$. With this general approach, standard finite element approximation spaces can be enriched with the introduction of additional functions in spaces $V_{i}$. The choice of these functions is based on an a priori knowledge of the solution or on numerical/analytical solutions of auxiliary problems. Several extension of the finite element method based on this construction have been proposed: X-FEM [22, 39, 38], GFEM [40].

\subsection{Construction of approximation space in $H^{1}(\Omega) \otimes L^{2}\left(\boldsymbol{\Xi}, d P_{\boldsymbol{\xi}}\right)$}

We now focus on the construction of an approximation space in $H^{1}(\Omega) \otimes L^{2}\left(\boldsymbol{\Xi}, d P_{\boldsymbol{\xi}}\right)$. Following the previous section, we define a partition of unity on $L^{2}\left(\boldsymbol{\Xi}, d P_{\boldsymbol{\xi}}\right)$ by introducing an open cover $\left\{\boldsymbol{\Xi}^{j}\right\}_{j \in J}$ of $\boldsymbol{\Xi}$ and introduce a set of functions $\left\{\chi_{j}\right\}_{j \in J}$ verifying the following properties (partition of unity):

$$
\operatorname{supp}\left(\chi_{j}\right) \subset \boldsymbol{\Xi}^{j}, \quad \sum_{j \in J} \chi_{j}=1 \text { on } \boldsymbol{\Xi}
$$

Let $M=\{(i, j), i \in I, j \in J\}$ and let us define the cover $\left\{\Upsilon_{m}\right\}_{m \in M}$ of $\Omega \times \boldsymbol{\Xi}$, with $\Upsilon_{(i, j)}=\Omega_{i} \times \boldsymbol{\Xi}^{j}$. Functions $\left\{\Phi_{m}\right\}_{m \in M}$, defined by $\Phi_{(i, j)}(\boldsymbol{x}, \boldsymbol{\xi})=\varphi_{i}(\boldsymbol{x}) \chi_{j}(\boldsymbol{\xi})$, form a partition of unity associated with the cover $\left\{\Upsilon_{m}\right\}_{m \in M}$ :

$$
\operatorname{supp}\left(\Phi_{m}\right) \subset \Upsilon_{m}, \quad \sum_{m \in M} \Phi_{m}=1 \text { on } \Upsilon
$$

An approximation space $W$ of $H^{1}(\Omega) \otimes L^{2}\left(\boldsymbol{\Xi}, d P_{\boldsymbol{\xi}}\right)$ can then be defined by

$$
W=\sum_{m \in M} \Phi_{m} W_{m}=\sum_{i \in I, j \in J} \varphi_{i} \chi_{j} W_{(i, j)}
$$

where the $W_{(i, j)} \subset H^{1}\left(\Omega_{i} \cap \Omega\right) \otimes L^{2}\left(\boldsymbol{\Xi} \cap \boldsymbol{\Xi}^{j}, d P_{\boldsymbol{\xi}}\right)$ are function spaces to be chosen. A simple choice for the $\chi_{j}$ consists in taking the (weighted) indicator functions of open subsets $\boldsymbol{\Xi}^{j}$. Let $\mathbb{Q}_{p}\left(\boldsymbol{\Xi}^{j}\right)=\operatorname{span}\left\{\boldsymbol{\xi}^{\alpha}=\prod_{k=1}^{m} \xi_{k}^{\alpha_{k}} ; \alpha \in \mathbb{N}^{m},\|\alpha\|_{\infty} \leqslant p\right\}\left(\right.$ resp. $\mathbb{P}_{p}\left(\boldsymbol{\Xi}^{j}\right)=\operatorname{span}\left\{\boldsymbol{\xi}^{\alpha}=\right.$ $\left.\left.\prod_{k=1}^{m} \xi_{k}^{\alpha_{k}} ; \alpha \in \mathbb{N}^{m},\|\alpha\|_{1} \leqslant p\right\}\right)$ be the spaces of multidimensional polynomial functions on $\boldsymbol{\Xi}^{j}$ with partial (resp. total) degree $p$. Classical spectral stochastic approximation spaces can then be obtained for different choices of covers $\left\{\boldsymbol{\Xi}^{j}\right\}_{j \in J}$ and spaces $W_{(i, j)}$ : 
- Non-overlapping cover $\left\{\boldsymbol{\Xi}^{j}\right\}_{j \in J}$ and $W_{(i, j)}=\mathbb{Q}_{q}\left(\Omega_{i}\right) \otimes \mathbb{Q}_{p}\left(\boldsymbol{\Xi}^{j}\right)$ (or $W_{(i, j)}=\mathbb{Q}_{q}\left(\Omega_{i}\right) \otimes$ $\left.\mathbb{P}_{p}\left(\boldsymbol{\Xi}^{j}\right)\right)$. $W$ corresponds to the tensorization of a finite element approximation space of degree $q+1$ in $H^{1}(\Omega)$ and of a finite element space of partial (or total) degree $p$ in $L^{2}\left(\boldsymbol{\Xi}, d P_{\boldsymbol{\xi}}\right)[30,32]$. The case $q=0$ corresponds to a classical linear finite element space in $H_{1}(\Omega)$.

- Overlapping cover $\left\{\boldsymbol{\Xi}^{j}\right\}_{j \in J}$ composed of the union of hierarchical binary partitions of $\boldsymbol{\Xi}$ and $W_{(i, j)}=\mathbb{Q}_{0}\left(\Omega_{i}\right) \otimes \mathbb{P}_{p}\left(\boldsymbol{\Xi}^{j}\right)$. W corresponds to the tensorization of a classical linear finite element space in $H^{1}(\Omega)$ and of a space of multi-wavelets of degree $p$ in $L^{2}\left(\boldsymbol{\Xi}, d P_{\boldsymbol{\xi}}\right)$ [41].

- Trivial cover $\{\boldsymbol{\Xi}\}$ and $W_{i}=\mathbb{Q}_{0}\left(\Omega_{i}\right) \otimes \mathbb{P}_{p}(\boldsymbol{\Xi})$. $W$ corresponds to the tensorization of a classical linear finite element space in $H^{1}(\Omega)$ and of a polynomial space of total degree $p$ in $L^{2}\left(\boldsymbol{\Xi}, d P_{\boldsymbol{\xi}}\right)$ (generalized polynomial chaos).

Non classical (enriched) approximation spaces can then be defined by introducing in function spaces $W_{(i, j)}$ suitable additional functions. These functions are usually chosen in order to capture irregularities in the solution which are not captured by classical polynomial spaces.

\section{ENRICHED SPECTRAL STOCHASTIC FINITE ELEMENT APPROXIMATION FOR THE CASE OF A RANDOM MATERIAL INTERFACE}

In this section, we detail the enrichment procedure of approximation spaces for the case of random material interfaces. Different types of enrichment are then introduced and discussed.

\subsection{Enriched approximation space}

An approximation space $W \subset H^{1}(\Omega) \otimes L^{2}\left(\boldsymbol{\Xi}, d P_{\boldsymbol{\xi}}\right)$ is here constructed by using the partition of unity method. In the following, we use a trivial partition $\{\boldsymbol{\Xi}\}$ at stochastic level and we identify the set $M$ with $I$. A function $u \in W$ will then be written

$$
u(\boldsymbol{x}, \boldsymbol{\xi})=\sum_{i \in I} \varphi_{i}(\boldsymbol{x}) u_{i}(\boldsymbol{x}, \boldsymbol{\xi}), \quad u_{i} \in W_{i}
$$

For the problem with a random material interface, the idea is to introduce in some particular spaces $W_{i}$ additional functions allowing to capture the irregularity of the solution $u(\boldsymbol{x}, \boldsymbol{\xi})$ with respect to $\boldsymbol{x}$ and $\boldsymbol{\xi}$. By drawing inspiration of enrichment procedures in the deterministic framework $[23,38]$, we propose the following definition of function spaces $W_{i}$ :

$$
\begin{aligned}
& W_{i}=\mathbb{P}_{p}(\boldsymbol{\Xi}), \quad i \in I \backslash I^{+} \\
& W_{i}=\mathbb{P}_{p}(\boldsymbol{\Xi})+\psi(\boldsymbol{x}, \boldsymbol{\xi}) \mathbb{P}_{p}(\boldsymbol{\Xi}), \quad i \in I^{+}
\end{aligned}
$$

where $I^{+}$denotes a subset of enriched supports $\Omega_{i}$ and where $\psi$ is an enrichment function for which we will discuss different choices in section 6.4 . In the following, we denote $\mathcal{S}_{P}:=\mathbb{P}_{p}(\boldsymbol{\Xi})$ the space of polynomial functions with total degree $p$. An approximation $u \in W$ can then be written in the following form

$$
u=\sum_{i \in I} \varphi_{i}(\boldsymbol{x}) a_{i}(\boldsymbol{\xi})+\sum_{i \in I^{+}} \varphi_{i}(\boldsymbol{x}) \psi(\boldsymbol{x}, \boldsymbol{\xi}) a_{i}^{+}(\boldsymbol{\xi}), \quad a_{i}, a_{i}^{+} \in \mathcal{S}_{P}
$$


Let us note that for a given outcome $\boldsymbol{\xi}$, equation (33) corresponds to the enrichment proposed in the deterministic X-FEM framework [23, 38]. Let us now introduce an orthonormal basis $\left\{H_{\alpha}\right\}_{\alpha=1}^{P}$ of $\mathcal{S}_{P}$ (generalized polynomial chaos basis). An approximation $u \in W$ is then written

$$
u=\sum_{i \in I} \sum_{\alpha=1}^{P} \varphi_{i}(\boldsymbol{x}) H_{\alpha}(\boldsymbol{\xi}) a_{i, \alpha}+\sum_{i \in I^{+}} \sum_{\alpha=1}^{P} \varphi_{i}(\boldsymbol{x}) \psi(\boldsymbol{x}, \boldsymbol{\xi}) H_{\alpha}(\boldsymbol{\xi}) a_{i, \alpha}^{+}, \quad a_{i, \alpha}, a_{i, \alpha}^{+} \in \mathbb{R}
$$

The corresponding enriched approximation space in $\mathcal{W}=\mathcal{V} \otimes \mathcal{S}$ is then defined by

$$
\mathcal{W}_{h, P}^{+}=\left\{v \in W ; v_{\mid \Gamma_{1}}=0\right\}
$$

where ${ }^{+}$indicates that the approximation space is enriched.

Remark 3. Let us note that the enriched approximation space $\mathcal{W}_{h, P}^{+}$does not have a tensor product structure. Degrees of freedom $a_{i}$ and $a_{i}^{+}$must then be manipulated with caution. For example, the expectation of the solution field $u\left(\boldsymbol{x}_{i}, \boldsymbol{\xi}\right)$ at a given node $\boldsymbol{x}_{i}$ is not obtained by $E\left(u\left(\boldsymbol{x}_{i}, \boldsymbol{\xi}\right)\right)=E\left(a_{i}(\boldsymbol{\xi})\right)+E\left(a_{i}^{+}(\boldsymbol{\xi}) \phi\left(\boldsymbol{x}_{i}, \boldsymbol{\xi}\right)\right)$, which is not directly related to the expectation of the degrees of freedom.

\subsection{Galerkin approximation $\left(X-S F E M-G^{+}\right)$}

The galerkin approximation $u_{h, P} \in \mathcal{W}_{h, P}^{+}$is simply defined by

$$
A\left(u_{h, P}, v_{h, P}\right)=B\left(v_{h, P}\right) \quad \forall v_{h, P} \in \mathcal{W}_{h, P}^{+}
$$

We introduce the following notation for a function $u \in \mathcal{W}_{h, P}^{+}$:

$$
u(\boldsymbol{x}, \boldsymbol{\xi})=\sum_{i=1}^{N_{1}} \varphi_{\sigma_{1}(i)}(\boldsymbol{x}) a_{i}(\boldsymbol{\xi})+\sum_{i=1}^{N_{2}} \varphi_{\sigma_{2}(i)}(\boldsymbol{x}) \psi(\boldsymbol{x}, \boldsymbol{\xi}) a_{i}^{+}(\boldsymbol{\xi}), \quad a_{i}, a_{i}^{+} \in \mathcal{S}_{P}
$$

where degrees of freedom are ordered using suitable mappings $\sigma_{1}:\left\{1 \ldots N_{1}\right\} \rightarrow I$ and $\sigma_{2}:\left\{1 \ldots N_{2}\right\} \rightarrow I$. Let $\mathbf{a} \in \mathbb{R}^{N_{1}} \otimes \mathcal{S}_{P}$ and $\mathbf{a}^{+} \in \mathbb{R}^{N_{2}} \otimes \mathcal{S}_{P}$ be the random vectors gathering the degrees of freedom $\left\{a_{i}\right\}$ and $\left\{a_{i}^{+}\right\}$. Let $\mathbf{u}=\left(\mathbf{a} \mathbf{a}^{+}\right) \in \mathbb{R}^{N} \otimes \mathcal{S}_{P}$, with $N=N_{1}+N_{2}$. Equation (35) then corresponds to the equation (16) for the random vector $\mathbf{u} \in \mathbb{R}^{N} \otimes \mathcal{S}_{P}$, where the random matrix $\mathbf{A}$ and random vector $\mathbf{b}$ are defined by

$$
\mathbf{A}=\left(\begin{array}{cc}
\mathbf{A}_{a a} & \mathbf{A}_{a a^{+}} \\
\mathbf{A}_{a^{+} a} & \mathbf{A}_{a^{+} a^{+}}
\end{array}\right), \quad \mathbf{b}=\left(\begin{array}{c}
\mathbf{b}_{a} \\
\mathbf{b}_{a^{+}}
\end{array}\right)
$$

with

$$
\begin{aligned}
& \left(\mathbf{A}_{a a}\right)_{i j}=\int_{\Omega} \nabla \varphi_{\sigma_{1}(i)} \cdot\left(\kappa_{1} \mathcal{H}(-\phi)+\kappa_{2} \mathcal{H}(\phi)\right) \cdot \nabla \varphi_{\sigma_{1}(j)} d x \\
& \left(\mathbf{A}_{a a^{+}}\right)_{i j}=\left(\mathbf{A}_{a^{+} a}\right)_{j i}=\int_{\Omega} \nabla \varphi_{\sigma_{1}(i)} \cdot\left(\kappa_{1} \mathcal{H}(-\phi)+\kappa_{2} \mathcal{H}(\phi)\right) \cdot \nabla\left(\varphi_{\sigma_{2}(j)} \psi\right) d x \\
& \left(\mathbf{A}_{a^{+} a^{+}}\right)_{i j}=\int_{\Omega} \nabla\left(\varphi_{\sigma_{2}(i)} \psi\right) \cdot\left(\kappa_{1} \mathcal{H}(-\phi)+\kappa_{2} \mathcal{H}(\phi)\right) \cdot \nabla\left(\varphi_{\sigma_{2}(j)} \psi\right) d x \\
& \left(\mathbf{b}_{a}\right)_{i}=\int_{\Omega} \varphi_{\sigma_{1}(i)} f d x+\int_{\Gamma_{2}} \varphi_{\sigma_{1}(i)} g d x \\
& \left(\mathbf{b}_{a^{+}}\right)_{i}=\int_{\Omega} \varphi_{\sigma_{2}(i)} \psi f d x+\int_{\Gamma_{2}} \varphi_{\sigma_{2}(i)} \psi g d x
\end{aligned}
$$


From a computational point of view, the solution of equation (16) requires the same ingredients as the one described in section 4.2.2: suitable stochastic integration of finite element contributions $E\left(\mathbf{A}_{K} H_{\alpha} H_{\beta}\right)$ and $E\left(\mathbf{b}_{K} H_{\alpha}\right)$ and assembly of these element quantities. Let us note that even if sources $f$ and $g$ are deterministic, $\mathbf{b}_{a^{+}}$can be random due to the presence of function $\psi$.

\section{3. $L^{2}$ projection $\left(X-S F E M-P^{+}\right)$}

The approximate solution $u_{h, P} \in \mathcal{W}_{h, P}^{+}$can also be defined by using a $L^{2}$ projection at stochastic level. The proposed $L^{2}$ projection method consists in defining the approximation $u_{h, P} \in \mathcal{W}_{h, P}^{+}$by computing the $L^{2}$ projection of the random vector $\mathbf{u} \in \mathbb{R}^{N} \otimes \mathcal{S}$ (semidiscretized solution) onto the subspace $\mathbb{R}^{N} \otimes \mathcal{S}_{P}$. Computational aspects are the same as in section 4.3: computing the coefficients of the decomposition $\mathbf{u}=\sum_{\alpha=1}^{P} \mathbf{u}_{\alpha} H_{\alpha}$ requires the solution of independent deterministic problems (25), which correspond to systems of equations obtained with a deterministic eXtended Finite Element Method (for different outcomes $\boldsymbol{\xi}=\mathbf{y}_{q}$ of the geometry).

\subsection{Choices of enrichment functions}

The question is now: how to choose the enrichment function $\psi$ and the set $I^{+}$of enriched supports? In this section, we discuss different choices. We illustrate these choices on a simple example. We consider a domain $\Omega=(0,1) \times(0,1)$ and a random material interface defined by the iso-zero of the level-set $\phi(\boldsymbol{x}, \xi)=x-\xi$, where $\xi \in U(0.3,0.7)$ is a uniform random variable $^{\ddagger}$ (see figure 1 ).

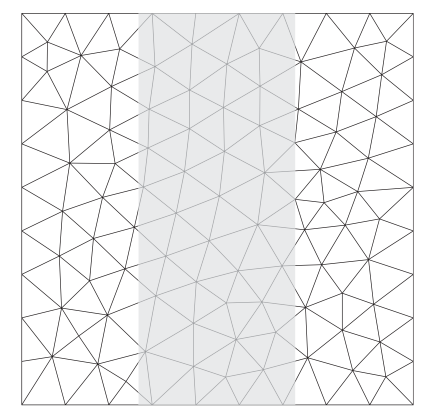

Figure 1. Illustration : mesh of $\Omega=[0,1] \times[0,1]$, vertical material interface given by $\phi=x-\xi=0$ with $\xi \in U(0.3,0.7)$

For each choice of enrichment, we define the set of enriched elements $\mathcal{T}_{h}^{+} \subset \mathcal{T}_{h}$, which have a non-zero contribution in the blocks of matrix $\mathbf{A}$ and vector $\mathbf{b}$ which are associated with the enriched degrees of freedom. $\mathcal{T}_{h}^{+}$is composed by the elements contained in the enriched supports $\left\{\Omega_{i}\right\}_{i \in I^{+}}$and which are possibly intersecting the spatial support of function $\psi(\cdot, \boldsymbol{\xi})$ :

$$
\mathcal{T}_{h}^{+}=\left\{K \in \mathcal{T}_{h} ; K \subset \cup_{i \in I^{+}} \Omega_{i}, P_{\boldsymbol{\xi}}(K \cap \operatorname{supp}(\psi(\cdot, \boldsymbol{\xi})) \neq \emptyset)>0\right\}
$$

\section{Choice 0}

${ }^{\ddagger} \xi \in U(a, b)$ denotes a uniform random variable with values in $(a, b)$ 
The first choice consists in taking for the function $\psi$ the absolute value of the level-set, i.e. $\psi=|\phi|$. This function has the desired irregularities at space level (for a given $\boldsymbol{\xi}$, discontinuity of the gradients across $\Gamma(\boldsymbol{\xi})$ ) and stochastic level (for a given $\boldsymbol{x}$, irregularity with respect to $\boldsymbol{\xi})$. This function can be written:

$$
\psi(\boldsymbol{x}, \boldsymbol{\xi})=|\phi(\boldsymbol{x}, \boldsymbol{\xi})|=\left\{\begin{array}{ccc}
-\sum_{i \in I} \varphi_{i}(\boldsymbol{x}) \phi_{i}(\boldsymbol{\xi}) & \text { if } & \boldsymbol{x} \in \Omega^{1}(\boldsymbol{\xi}) \\
\sum_{i \in I} \varphi_{i}(\boldsymbol{x}) \phi_{i}(\boldsymbol{\xi}) & \text { if } & \boldsymbol{x} \in \Omega^{2}(\boldsymbol{\xi})
\end{array}\right.
$$

The set of the enriched supports can be chosen as:

$$
I^{+}=\left\{i \in I ; P_{\boldsymbol{\xi}}\left(\Omega_{i} \cap \Gamma(\boldsymbol{\xi}) \neq \emptyset\right)>0\right\}
$$

which is the set of supports $\Omega_{i}$ which have a non-zero probability to be cut by the interface (Figure 2). Figure 3 illustrates several outcomes of the function $\psi$. In the deterministic framework, it has been numerically proved [38] that this enrichment function does not allow to obtain an optimal convergence of the approximation $\left(O\left(h^{2}\right)\right.$ convergence rate with respect to the $L^{2}$ norm). This choice will not be considered in the numerical examples.

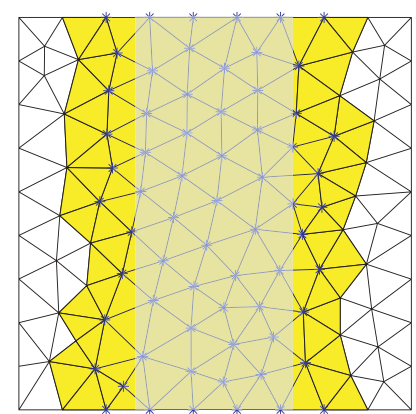

Figure 2. Illustration of choice 0: set of enriched nodes $I^{+}(*)$ and enriched finite elements $K \in \mathcal{T}_{h}^{+}$
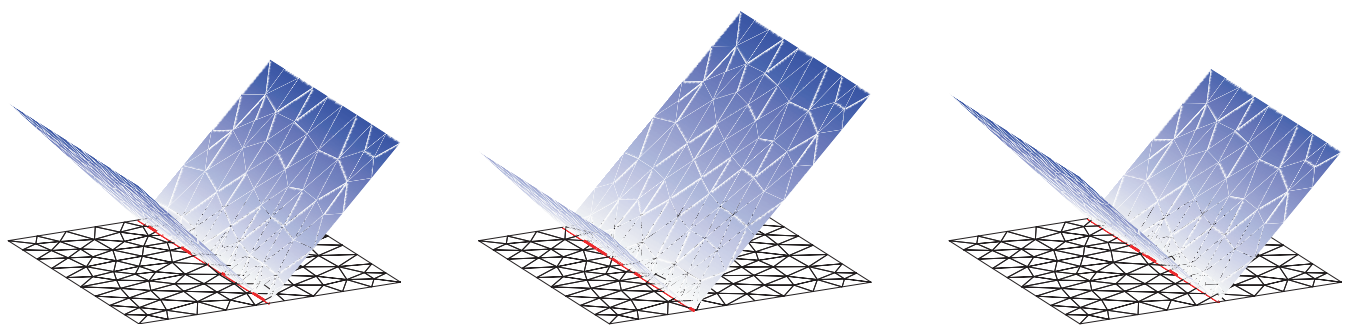

Figure 3. Illustration of choice 0: 4 outcomes of function $\psi$

Choice 1 Another possible choice, inspired from [38], consists in taking

$$
\psi(\boldsymbol{x}, \boldsymbol{\xi})=\sum_{i \in I} \varphi_{i}(\boldsymbol{x})\left|\phi_{i}(\boldsymbol{\xi})\right|-|\phi(\boldsymbol{x}, \boldsymbol{\xi})|=\left\{\begin{array}{lll}
\sum_{i \in I} \varphi_{i}(\boldsymbol{x})\left(\left|\phi_{i}(\boldsymbol{\xi})\right|+\phi_{i}(\boldsymbol{\xi})\right) & \text { if } & \boldsymbol{x} \in \Omega^{1}(\boldsymbol{\xi}) \\
\sum_{i \in I} \varphi_{i}(\boldsymbol{x})\left(\left|\phi_{i}(\boldsymbol{\xi})\right|-\phi_{i}(\boldsymbol{\xi})\right) & \text { if } & \boldsymbol{x} \in \Omega^{2}(\boldsymbol{\xi})
\end{array}\right.
$$


The set of enriched supports $I^{+}$is still defined by (43). We notice a special feature of this enrichment: for a given $\boldsymbol{\xi}$, the function $\psi(\boldsymbol{x}, \boldsymbol{\xi})$ takes only non-zero values on the finite elements cut by the interface (see the set of enriched finite elements $\mathcal{T}_{h}^{+}$on Figure 4 and samples of $\psi$ on Figure 5). In the deterministic framework [38], it has been shown that this function allows to recover the optimal convergence rate obtained with a finite element approximation associated with a mesh conforming the interface $\left(O\left(h^{2}\right)\right.$ convergence rate with respect to the $L^{2}$ norm). However, in the stochastic framework, this enrichment presents some drawbacks for the computation of the Galerkin projection. Indeed, for an element $K \in \mathcal{T}_{h}^{+}$, the computation of the finite element contribution $E\left(\mathbf{A}_{K} H_{\alpha} H_{\beta}\right)$ requires a stochastic integration of a function which depends on the restriction $\psi(\boldsymbol{x}, \boldsymbol{\xi})$ to the element $K$, denoted $\psi_{K}: K \times \boldsymbol{\Xi} \rightarrow \mathbb{R}$. The support of function $\psi_{K}$ appears to be a small subset of $K \times \boldsymbol{\Xi}$, corresponding to outcomes $\boldsymbol{\xi} \in \boldsymbol{\Xi}$ such that $K \cap \Gamma(\boldsymbol{\xi}) \neq \emptyset$. In the case where the range of variation of $\Gamma$ is large (i.e. $\Gamma$ spans "several elements layers"), we have to perform a stochastic integration of a function with a low probability to differ from zero. The integration procedure proposed in [21] is still efficient but requires a high level of accuracy, which generates high computational costs. Another drawback of this choice is that the degrees of freedom $\mathbf{a}(\boldsymbol{\xi})$ and $\mathbf{a}^{+}(\boldsymbol{\xi})$ are non-smooth functions of $\boldsymbol{\xi}$. Then, the projection of these degrees of freedom on a polynomial stochastic basis leads to a poor accuracy and bad convergence properties (for Galerkin and $L^{2}$ projections). This enrichment function will be denoted by $\psi_{1}$ and will be tested in the numerical examples in Section 7 .

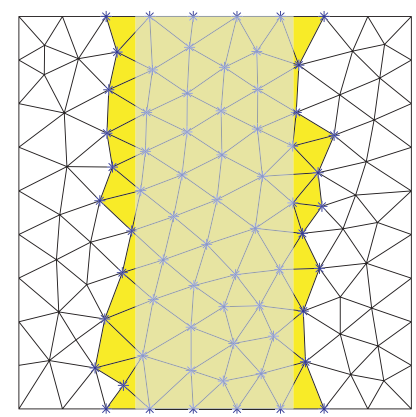

Figure 4. Illustration of choice 1: set of enriched nodes $I^{+}(*)$ and enriched finite elements $K \in \mathcal{T}_{h}^{+}$.
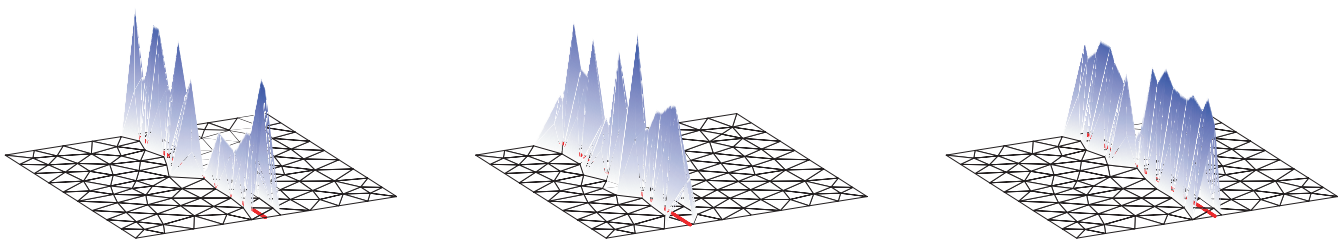

Figure 5. Illustration of choice 1: 4 outcomes of function $\psi$ 
Choice 2 As a final choice, we propose to define

$$
\psi(\boldsymbol{x}, \boldsymbol{\xi})=\left\{\begin{array}{lll}
\sum_{i \in I_{1}^{+}} \varphi_{i}(\boldsymbol{x})\left(\beta+\phi_{i}(\boldsymbol{\xi})\right) & \text { if } & \boldsymbol{x} \in \Omega^{1}(\boldsymbol{\xi}) \\
\sum_{i \in I_{1}^{+}} \varphi_{i}(\boldsymbol{x})\left(\beta-\phi_{i}(\boldsymbol{\xi})\right) & \text { if } & \boldsymbol{x} \in \Omega^{2}(\boldsymbol{\xi})
\end{array}\right.
$$

where $\beta$ is a constant. The set of enriched supports is chosen as $I^{+}=I_{1}^{+} \cup I_{2}^{+}$, with

$$
\begin{aligned}
& I_{1}^{+}=\left\{i \in I ; P_{\boldsymbol{\xi}}\left(\Omega_{i} \cap \Gamma(\boldsymbol{\xi}) \neq \emptyset\right)>0\right\} \\
& I_{2}^{+}=\left\{i \in I \backslash I_{1}^{+} ; \exists j \in I_{1}^{+}, \boldsymbol{x}_{i} \in \partial \Omega_{j}\right\}
\end{aligned}
$$

$I_{1}^{+}$is then the set of nodes whose support is possibly cut by the interface (corresponding to the sets $I^{+}$of choices 0 and 1 ) and $I_{2}^{+}$is a complementary set of nodes connected to supports $\left\{\Omega_{i}\right\}_{i \in I_{1}^{+}}$(see figure 6). In fact, the proposed function $\psi$ is a modification of the function $(\beta-|\phi|)$ which consists in restricting its expansion on the interpolation basis $\left\{\varphi_{i}\right\}$ to the set $i \in I_{1}^{+}$. For a given $\boldsymbol{\xi}$, this choice allows to obtain a spatial support of $\psi(\cdot, \boldsymbol{\xi})$ which is $\cup_{i \in I_{1}^{+}} \Omega_{i}$, i.e. the union of supports cut by the interface (see figure 7 ). In practise, parameter $\beta$ is chosen in the following way:

$$
\beta \approx \sup _{i \in I^{+}, \boldsymbol{\xi} \in \boldsymbol{\Xi}}\left|\phi_{i}(\boldsymbol{\xi})\right|
$$

We observe that this choice allows to improve the condition number of the discretized problem (19) (in particular when comparing with $\beta=0$ ). Numerical experiments of the authors, which are not illustrated here, have shown that this choice of $\psi$ keeps the good convergence properties of choice 1 in the deterministic framework. In the stochastic framework, this choice allows to circumvent the drawbacks of choice 1 . The stochastic integration is made easier by the fact that the support of function $\psi_{K}$, for $K \in \mathcal{T}_{h}^{+}$, is the entire domain $K \times \boldsymbol{\Xi}$. Moreover, degrees of freedom $\mathbf{a}$ and $\mathbf{a}^{+}$recover a good regularity at stochastic level, which leads to a good accuracy and convergence properties when using a standard global polynomial approximation at the stochastic level. This enrichment function will be denoted by $\psi_{2}$ in Section 7 .

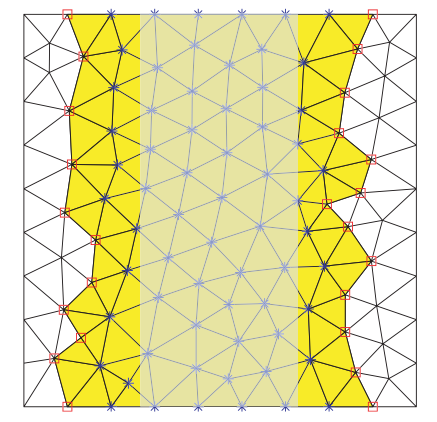

Figure 6. Illustration of choice 2: sets of enriched nodes $I_{1}^{+}(*)$ and $I_{2}^{+}(\square)$ and enriched finite elements $K \in \mathcal{T}_{h}^{+}$ 

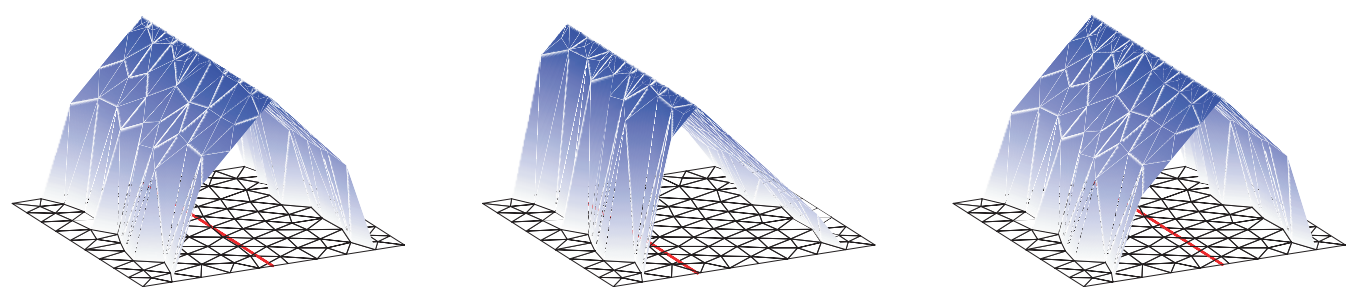

Figure 7. Illustration of choice 2: 4 outcomes of function $\psi$

\section{NUMERICAL EXAMPLES}

In this section, we illustrate the proposed method on three numerical examples in linear elasticity. These examples will allow to compare the accuracy of the different enrichment strategies and of the different projection techniques (Galerkin or $L^{2}$ projections).

\subsection{Preliminaries}

7.1.1. Elasticity problem We here apply the method to the analysis of the deformations of a structure under small perturbations assumption. The structure is composed by two linear elastic homogenous and isotropic materials, separated by a random interface $\Gamma(\boldsymbol{\xi})=$ $\partial \Omega^{1}(\boldsymbol{\xi}) \cap \partial \Omega^{2}(\boldsymbol{\xi})$. We denote by $\boldsymbol{u} \in \mathcal{W}=\mathcal{V} \otimes \mathcal{S}$ the displacement field, with $\mathcal{V}=\{\boldsymbol{v} \in$ $\left.\left.\left(H_{1}(\Omega)\right)^{d}\right) ; \boldsymbol{v}_{\mid \Gamma}=0\right\}$. The weak formulation writes as in equation (4), with the following definition of bilinear and linear forms:

$$
\begin{aligned}
& A(\boldsymbol{u}, \boldsymbol{v})=\int_{\boldsymbol{\Xi}} \int_{\Omega} \boldsymbol{\epsilon}(\boldsymbol{v}):\left(\boldsymbol{C}_{1} \mathcal{H}(-\phi)+\boldsymbol{C}_{2} \mathcal{H}(\phi)\right): \boldsymbol{\epsilon}(\boldsymbol{u}) d x d P_{\boldsymbol{\xi}} \\
& B(\boldsymbol{v})=\int_{\boldsymbol{\Xi}} \int_{\Gamma_{2}} \boldsymbol{v} \cdot \boldsymbol{F} d s d P_{\boldsymbol{\xi}}
\end{aligned}
$$

where $\boldsymbol{C}_{1}$ and $\boldsymbol{C}_{2}$ are the Hooke elasticity tensors of the two phases of the material, $\boldsymbol{\epsilon}(\boldsymbol{u})$ is the strain tensor (symmetric part of the gradient of $\boldsymbol{u}$ ) and $\boldsymbol{F}$ is a surface load applied on a part $\Gamma_{2}$ of the boundary.

7.1.2. Approximation spaces and projection techniques Approximation space $\mathcal{W}_{h, P}^{+}$is simply defined by using definition (34) for each component of the displacement field, i.e.

$$
\boldsymbol{u}_{h, P}=\sum_{i \in I} \varphi_{i}(\boldsymbol{x}) \boldsymbol{a}_{i}(\boldsymbol{\xi})+\sum_{i \in I^{+}} \varphi_{i}(\boldsymbol{x}) \psi(\boldsymbol{x}, \boldsymbol{\xi}) \boldsymbol{a}_{i}^{+}(\boldsymbol{\xi}), \quad \boldsymbol{a}_{i}, \boldsymbol{a}_{i}^{+} \in \mathbb{R}^{d} \otimes \mathcal{S}_{P}
$$

In the numerical examples, we choose for $\mathcal{S}_{P}=\mathbb{P}_{p}(\boldsymbol{\Xi})$ a polynomial space of degree $p$. We denote by X-SFEM-G and X-SFEM-P the approximate solutions respectively obtained with Galerkin and $L^{2}$ projections on the classical approximation space $\mathcal{W}_{h, P}$. We denote by $\mathrm{X}$ SFEM-G ${ }_{1}^{+}$and X-SFEM-P ${ }_{1}^{+}$(resp. X-SFEM-G $\mathrm{C}_{2}^{+}$and X-SFEM-P ${ }_{2}^{+}$) the approximate solutions obtained with Galerkin and $L^{2}$ projections on $\mathcal{W}_{h, P}^{+}$when using an enrichment strategy based on function $\psi_{1}$ (resp. $\psi_{2}$ ).

7.1.3. Error estimation In order to estimate the accuracy of the approximations, we introduce the following global error indicator between the approximate solution $\boldsymbol{u}_{h, P}(\boldsymbol{x}, \boldsymbol{\xi})$ and the exact 
solution $\boldsymbol{u}(\boldsymbol{x}, \boldsymbol{\xi})$ :

$$
\varepsilon_{h, p}=\frac{\left\|\boldsymbol{u}_{h, P}-\boldsymbol{u}\right\|_{L^{2}(\Omega \times \boldsymbol{\Xi})}}{\|\boldsymbol{u}\|_{L^{2}(\Omega \times \boldsymbol{\Xi})}}
$$

with

$$
\|\boldsymbol{u}\|_{L^{2}(\Omega \times \boldsymbol{\Xi})}^{2}=\int_{\boldsymbol{\Xi}} \int_{\Omega} \boldsymbol{u}(\boldsymbol{x}, \mathbf{y}) \cdot \boldsymbol{u}(\boldsymbol{x}, \mathbf{y}) d x d P_{\boldsymbol{\xi}}(\mathbf{y})
$$

We also define a local error indicator $\varepsilon_{h, p}^{K}$ which is the local contribution to the global $\operatorname{error}^{\S}$ :

$$
\varepsilon_{h, p}^{K}=\frac{\left\|\boldsymbol{u}_{h, P}-\boldsymbol{u}\right\|_{L^{2}(K \times \boldsymbol{\Xi})}}{\|\boldsymbol{u}\|_{L^{2}(\Omega \times \boldsymbol{\Xi})}} .
$$

\subsection{Example 1: random plate with random vertical material interface}

7.2.1. Problem definition We consider a square domain $\Omega=(0,1) \times(0,1) \subset \mathbb{R}^{2}$ composed of two materials separated by a random vertical interface $\Gamma(\xi)$, whose horizontal location depends on a single uniform random variable $\xi \in U(0.4,0.6)$ (see Figure 8). The interface $\Gamma(\xi)$ is characterized by the following level-set function: for $\boldsymbol{x}=(x, y) \in \Omega, \phi(\boldsymbol{x}, \xi)=x-\xi$. The problem is here formulated in a one dimensional probability space $\left(\Xi, \mathcal{B}_{\Xi}, P_{\xi}\right)$, with $\Xi=(0.4,0.6)$ and $P_{\xi}$ the uniform measure on $(0.4,0.6)$. The two materials have the following deterministic properties: Young modulus $E_{1}=1$ and Poisson coefficient $\nu_{1}=0$ in $\Omega^{1}(\xi)$, $E_{2}=2$ and $\nu_{2}=0$ in $\Omega^{2}(\xi)$. The plate is fixed on the left edge and submitted to a uniform tension load $\boldsymbol{F}=(1,0)$ on the right edge (see Figure 8 ). This example is in fact a onedimensional problem whose exact solution is known. For the approximation at space level, we

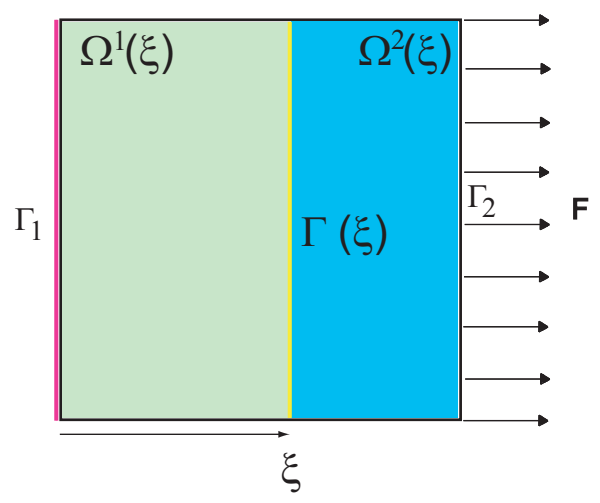

Figure 8. Example 1: plate in tension with random vertical material interface.

use the finite element mesh represented on Figure 6. At stochastic level, we use a generalized polynomial chaos with degree $p$ : basis functions $\left\{H_{\alpha}\right\}$ of $\mathcal{S}_{P}$ are the Legendre polynomials on $\Xi=(0.4,0.6)$.

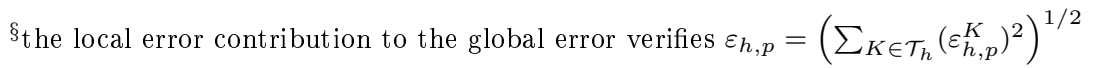


7.2.2. Accuracy of the X-SFEM-G approximation We first study the convergence of the different X-SFEM-G approximations. Table I indicates the global error indicator $\varepsilon_{h, p}$ (see equation (49)) according to polynomial chaos degree $p$.

\begin{tabular}{|c|c|c|c|c|}
\cline { 2 - 5 } \multicolumn{1}{c|}{} & $p=1$ & $p=2$ & $p=3$ & $p=4$ \\
\hline X-SFEM-G & $910^{-3}$ & $6.610^{-3}$ & $5.810^{-3}$ & $5.510^{-3}$ \\
\hline $\mathrm{X}_{\text {SFEMM-G }}^{+}$ & $510^{-3}$ & $210^{-3}$ & $910^{-4}$ & $5.710^{-4}$ \\
\hline $\mathrm{X}_{\mathrm{S} S F E M-G}{ }_{2}^{+}$ & $4.310^{-14}$ & $4.110^{-14}$ & $410^{-14}$ & $4.210^{-14}$ \\
\hline
\end{tabular}

Table I. Example 1 : global errors $\varepsilon_{h, p}$ obtained with X-SFEM-G, X-SFEM-G ${ }_{1}^{+}$and X-SFEM-G ${ }_{2}^{+}$, according to polynomial chaos degree $p$.

We observe that X-SFEM-G $\mathrm{G}_{2}^{+}$leads to the exact solution, while X-SFEM-G $\mathrm{C}_{1}^{+}$leads to a relatively good approximation but not to the exact one. However, X-SFEM-G $\mathrm{G}_{1}^{+}$systematically leads to a lower error than X-SFEM-G. In order to understand why the enrichment function $\psi_{1}$ does not allow to get the exact solution, we illustrate on Figure 9 the response surface of the horizontal displacement for a particular point $\boldsymbol{x}=(0.5,0.55)$ such that $P_{\xi}\left(\boldsymbol{x} \in \Omega^{1}(\xi)\right)>0$ and $P_{\xi}\left(\boldsymbol{x} \in \Omega^{2}(\xi)\right)>0$. This figure shows the response surfaces obtained with the three approximate solutions (X-SFEM-G, X-SFEM-G $\mathrm{G}_{1}^{+}$and X-SFEM-G ${ }_{2}^{+}$) and the exact solution. First, we observe that the $\mathrm{X}-\mathrm{SFEM}-\mathrm{G}$ approximation is not able to capture the irregularity

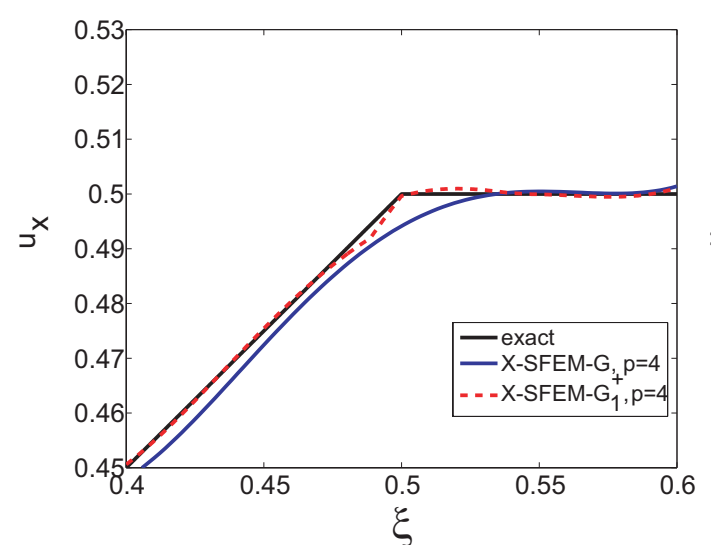

(a)

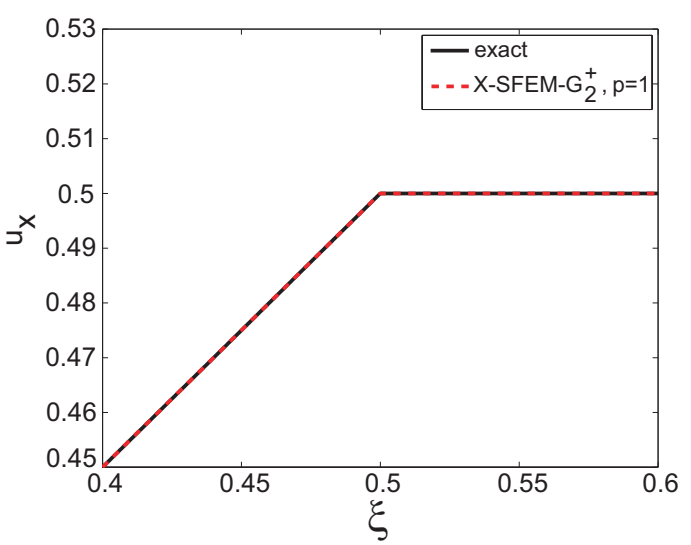

(b)

Figure 9. Example 1: response surfaces of horizontal displacement at point $\boldsymbol{x}=(0.5,0.55)$ : comparison between exact, X-SFEM-G and X-SFEM-G ${ }_{1}^{+}$solutions (a), comparison between exact and X-SFEM$\mathrm{G}_{2}^{+}$solutions (b).

with respect to $\xi$. With the X-SFEM-G $\mathrm{X}_{1}^{+}$approximation, this irregularity is captured but other spurious irregularities appear. With this choice of enrichment, degrees of freedom $\boldsymbol{a}_{i}(\xi)$ and $\boldsymbol{a}_{i}^{+}(\xi)$ are non-smooth functions of $\xi$ and are not well represented on a polynomial chaos basis. Figure 10 shows the response surfaces of an enriched degree of freedom obtained with the $\mathrm{X}$ SFEM-G ${ }_{1}^{+}$for different polynomial degrees $p$. On this figure, the reference response surface is obtained with a collection of deterministic X-FEM computations for different positions of the 
interface (with a $\psi_{1}$ enrichment). We notice that the enriched degree of freedom is piecewise constant and that the Galerkin projection only seeks to approximate the non-zero part of the enriched degree of freedom. For this problem, when the range of variation of $\Gamma(\xi)$ is increasing (more and more elements layers spanned by the interface), the accuracy of the X-SFEM-G solution decreases. On the other hand, the X-SFEM-G ${ }_{2}^{+}$perfectly matches the exact solution. The enrichment function $\psi_{2}$ captures the irregularity with respect to $\xi$ and degrees of freedom become smooth (polynomial) functions of $\xi$ which are exactly represented on a polynomial basis.

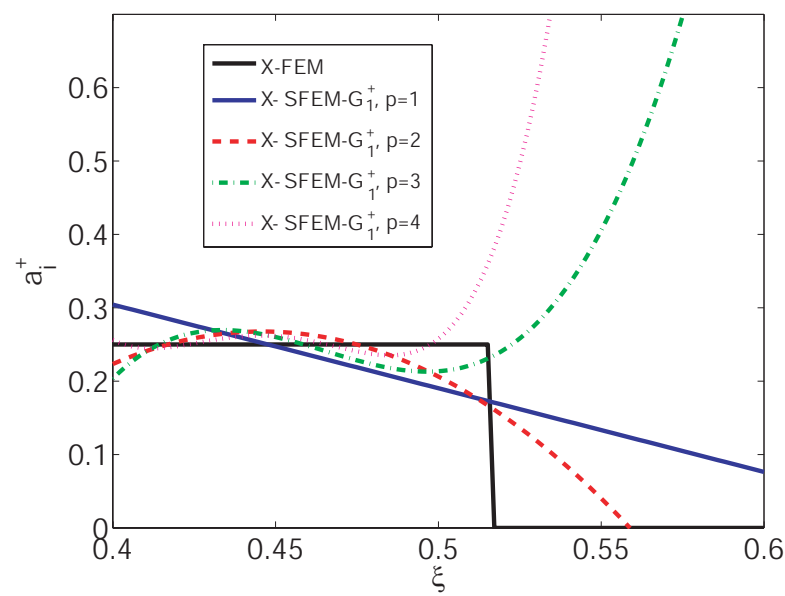

Figure 10. Example 1: response surfaces of an enriched degree of freedom obtain with X-SFEM-G ${ }_{1}^{+}$ for different polynomial chaos orders $p$.

7.2.3. Accuracy of the X-SFEM-P approximation We now focus on the results obtained with the $L^{2}$ projection method. Table II indicates the global error indicator $\varepsilon_{h, p}$ according to polynomial chaos degree $p$ for standard approximation X-SFEM-P and enriched approximations X-SFEM-P ${ }_{1}^{+}$and X-SFEM-P ${ }_{2}^{+}$based on enrichment functions $\psi_{1}$ and $\psi_{2}$ respectively. We notice that the results are close to those obtained with the Galerkin projection (see table I). The exact solution is only obtained with X-SFEM-P ${ }_{2}^{+}$.

\begin{tabular}{|c|c|c|c|c|}
\cline { 2 - 5 } \multicolumn{1}{c|}{} & $p=1$ & $p=2$ & $p=3$ & $p=4$ \\
\hline $\mathrm{X}_{\text {SSFEM-P }}$ & $5.510^{-3}$ & $5.310^{-3}$ & $5.210^{-3}$ & $5.210^{-3}$ \\
\hline $\mathrm{X}_{\text {-SFEM-P }}^{+}$ & $1.410^{-3}$ & $110^{-3}$ & $7.210^{-4}$ & $3.910^{-4}$ \\
\hline $\mathrm{X}_{1}$-SFEM-P \\
\hline
\end{tabular}

Table II. Example 1: global errors $\varepsilon_{h, p}$ obtained with solutions X-SFEM-P, X-SFEM-P ${ }_{1}^{+}$and X-FEM$\mathrm{P}_{2}^{+}$according to polynomial chaos degree $p$.

It should be noticed that the choice of the stochastic quadrature is essential with the X-SFEM-P ${ }_{1}^{+}$approximation. In fact, the enriched basis functions $\psi_{1}(\boldsymbol{x}, \xi) \varphi_{i}(\boldsymbol{x})$ and the associated degrees of freedom $\boldsymbol{a}_{i}^{+}(\xi)$ have localized supports in $\Xi$. For a given $\boldsymbol{x}$, this support 
may be small if the size of finite elements is small compared to the range of variation of $\Gamma(\xi)$. If one uses a coarse stochastic quadrature (few integration points), the projection of enriched degrees of freedom $E\left(\boldsymbol{a}_{i}^{+} H_{\alpha}\right)$ may be badly estimated or even equal to zero if no integration points are located in the support of $\xi \mapsto \boldsymbol{a}_{i}^{+}(\xi)$. This drawback is circumvented when using the enrichment function $\psi_{2}$. With this enrichment, the enriched degrees of freedom $\boldsymbol{a}_{i}^{+}(\xi)$ have for support the whole stochastic domain $\boldsymbol{\Xi}$ and their projection on the polynomial basis is well estimated by using relatively coarse stochastic quadrature.

Remark 4. Let us notice that with $X-S F E M-P_{2}^{+}$, a pre-processing is needed in order to define the enriched structure of the finite element model. Then, X-SFEM- $P_{2}^{+}$loses a part of the non-intrusive character usually associated with $L^{2}$ stochastic projection techniques.

\subsection{Example 2: circular plate with circular random inclusion}

7.3.1. Problem definition We consider the circular domain $\Omega \subset \mathbb{R}^{2}$ represented on Figure 11(a). The two materials are separated by a circular random interface $\Gamma(\xi)$ with center $(0,0)$ and random radius $\xi \in U(0.72,1.8)$ which is a uniform random variable. The external boundary $\Gamma_{1}=\partial \Omega$ is a circle with radius $b=2$. This example will illustrate the ability of the X-SFEM method to deal with a large range of variation of the random interface. The problem is here formulated in a one dimensional probability space $\left(\Xi, \mathcal{B}_{\Xi}, P_{\xi}\right)$, with $\Xi=(0.72,1.8)$ and $P_{\xi}$ the uniform measure on $(0.72,1.8)$. We denote by $\Omega^{1}(\xi)$ the internal domain and by $\Omega^{2}(\xi)$ the external domain. The material properties are deterministic on each subdomain: $E_{1}=1$, $\nu_{1}=0.25$ in $\Omega^{1}$ and $E_{2}=10, \nu_{2}=0.3$ in $\Omega^{2}$. We impose a linear displacement field $\boldsymbol{u}=\boldsymbol{x}$ on the boundary $\Gamma_{1}$. In practise, the problem is reformulated on function $\tilde{\boldsymbol{u}}=\boldsymbol{u}-\boldsymbol{x} \in \mathcal{W}$ with homogeneous Dirichlet boundary conditions on $\Gamma_{1}$.

At the space level, we use a finite element mesh composed of 3-nodes triangular finite elements. In order to study convergence properties of the approximations, we consider three different meshes with average element size $h \in\{0.18,0.09,0.06\}$. Figure 11(b) shows one of these meshes, whose elements are split into three groups: the first group $\left(e_{1}\right)$ gathers elements surely in $\Omega^{1}$, the second group $\left(e_{2}\right)$ gathers elements surely in $\Omega^{2}$ and the third group $\left(e_{c}\right)$ gathers elements possibly cut by the interface $\Gamma(\xi)$. At stochastic level, we use a generalized polynomial chaos with degree $p$ for which the basis functions of $\mathcal{S}_{P}$ are the Legendre polynomials on $\Xi=(0.72,1.8)$. This problem has an analytical solution (see [23]). It allows us to perform a convergence analysis.

7.3.2. Accuracy of the X-SFEM-G approximation For this example, we only use a Galerkin projection for the approximation (X-SFEM-G). Using the global and local error indicators defined in equations (49) and (51), we propose to study the convergence of the different $\mathrm{X}$ SFEM solutions (X-SFEM-G, X-SFEM-G $\mathrm{G}_{1}^{+}$and X-SFEM-G $\mathrm{G}_{2}^{+}$) according to polynomial chaos degree $p \in\{1, \ldots 7\}$ and mesh size $h \in\{0.18,0.09,0.06\}$. Figure 12 presents the convergence of the global error indicator $\varepsilon_{h, p}$ with $p$ for $h=0.09$. As expected, the X-SFEM solution leads to significant error values and presents a slow convergence with $p$. Although the $\mathrm{X}$ SFEM-G $\mathrm{G}_{1}^{+}$approximation provides better results, we can observe a slow convergence with $p$. Thus, with X-SFEM-G $\mathrm{G}_{1}^{+}$, a high polynomial chaos degree is usually required in order to get an accurate (converged) approximation. The X-SFEM-G $\mathrm{G}_{2}^{+}$solution provides the best results. The convergence with $p$ of $\varepsilon_{h, p}$ is very fast (error close to $10^{-3}$ with only $p=2$ ).

Figure 13 indicates the local errors for the X-SFEM-G ${ }_{1}^{+}$approximation with $p=7$ and 


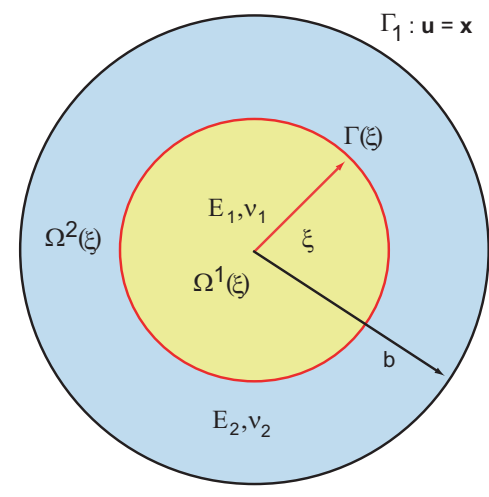

(a)

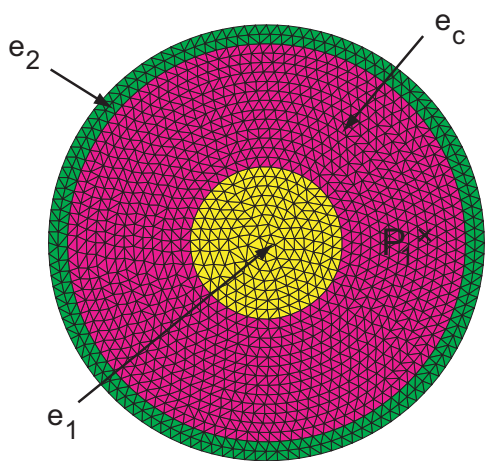

(b)

Figure 11. Example 2: circular plate with circular random inclusion (a) and X-SFEM mesh with three groups of elements (b).

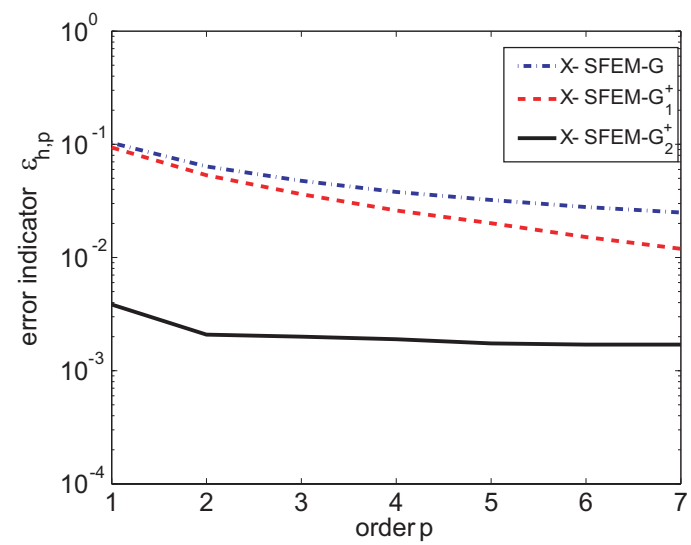

Figure 12. Example 2: convergence of error $\varepsilon_{h, p}$ according to $p$ for $h=0.09$ obtained with the three $\mathrm{X}-\mathrm{SFEM}-\mathrm{G}$ approximations.

the X-SFEM-G ${ }_{2}^{+}$approximation with $p=1$. We notice that with $\mathrm{X}-\mathrm{SFEM}-\mathrm{G}_{1}^{+}$with a high polynomial chaos degree, the local errors in finite elements belonging to the set $\left(e_{c}\right)$ are much higher than with X-SFEM-G $\mathrm{G}_{2}^{+}$with a very low polynomial chaos degree.

Figure 14 shows the convergence of $\varepsilon_{h, p}$ with the mesh size $h$ for a fixed polynomial chaos degree $p=3$. On this figure, only the $\mathrm{X}-\mathrm{SFEM}-\mathrm{G}_{2}^{+}$solution is represented. We can observe a good convergence rate $\left(O\left(h^{1.6}\right)\right)$ which is close to the optimal expected convergence rate (in $\left.O\left(h^{2}\right)\right)$.

Finally, we examine the response surfaces of the radial displacement obtained with the three X-SFEM approximations at a particular point $\boldsymbol{x}=(1.5,0)$ such that $P_{\xi}\left(\boldsymbol{x} \in \Omega^{1}(\xi)\right)>0$ and $P_{\xi}\left(\boldsymbol{x} \in \Omega^{2}(\xi)\right)>0$ (Point $P_{1}$ on Figure 11). Figure 15 illustrates these response surfaces. Two events $\Xi^{1} \in \mathcal{B}_{\Xi}$ and $\Xi^{2} \in \mathcal{B}_{\Xi}$ can be clearly distinguished, corresponding to outcomes $\xi$ such that $P_{1} \in \Omega^{1}(\xi)$ or $P_{1} \in \Omega^{2}(\xi)$ respectively. We first observe that on $\Xi^{2}$, the three approximate solutions give better response surfaces than on $\Xi^{1}$. This comes from properties of the Galerkin 


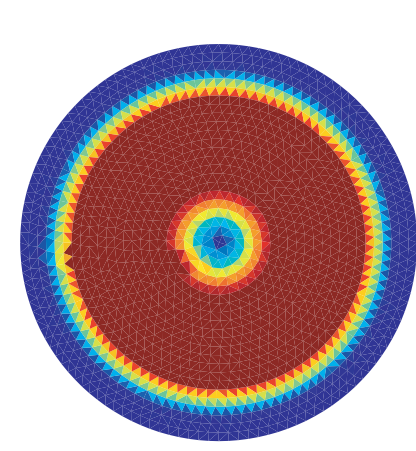

(a)

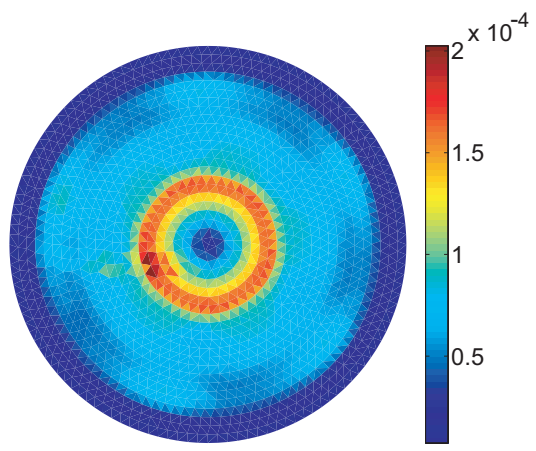

(b)

Figure 13. Example 2: local errors $\varepsilon_{h, p}^{K}$ obtained with X-SFEM-G ${ }_{1}^{+}$with $p=7$ (a) and X-SFEM-G ${ }_{2}^{+}$ with $p=1(\mathrm{~b})$.

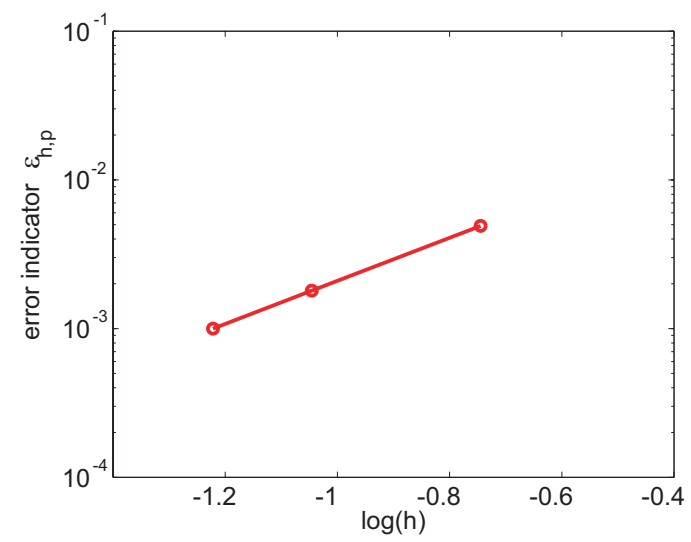

Figure 14. Example 2: convergence of error $\varepsilon_{h, p}$ according to $h$ obtained with X-SFEM-G ${ }_{2}^{+}$solution.

approximation. Roughly speaking, the Galerkin projection is associated with an inner product which gives a higher weight to outcomes $\xi$ in $\Xi^{2}$ (since $\frac{E_{2}}{E_{1}}=10$ ). However, on $\Xi^{1}$, the XSFEM-G ${ }_{2}^{+}$solution leads clearly to better results. We can notice that with X-SFEM-G $\mathrm{G}_{2}^{+}$, the irregularity is well captured by the enrichment function and a polynomial chaos of degree $p$ only equal to 1 is sufficient in order to accurately approximate the degrees of freedom.

\subsection{Example 3: homogenization of a cell with random inclusion}

7.4.1. Problem definition We consider a cell $\Omega=(0,1) \times(0,1)$ of an heterogeneous material composed of two material phases. The two phases $\Omega^{1}(\boldsymbol{\xi})$ and $\Omega^{2}(\boldsymbol{\xi})$ are separated by a random interface $\Gamma(\boldsymbol{\xi})$ characterized by the iso-zero of the following level-set:

$$
\begin{aligned}
& \phi(\boldsymbol{x}, \boldsymbol{\xi})=R(\alpha(\boldsymbol{x}), \boldsymbol{\xi})-\|\boldsymbol{x}-\boldsymbol{c}\|, \\
& \boldsymbol{c}=(0.5,0.5), \quad R(\alpha, \boldsymbol{\xi})=0.25+0.1\left(\xi_{1}(\boldsymbol{\xi}) \cos (2 \alpha)+\xi_{2}(\boldsymbol{\xi}) \sin (2 \alpha)\right)
\end{aligned}
$$




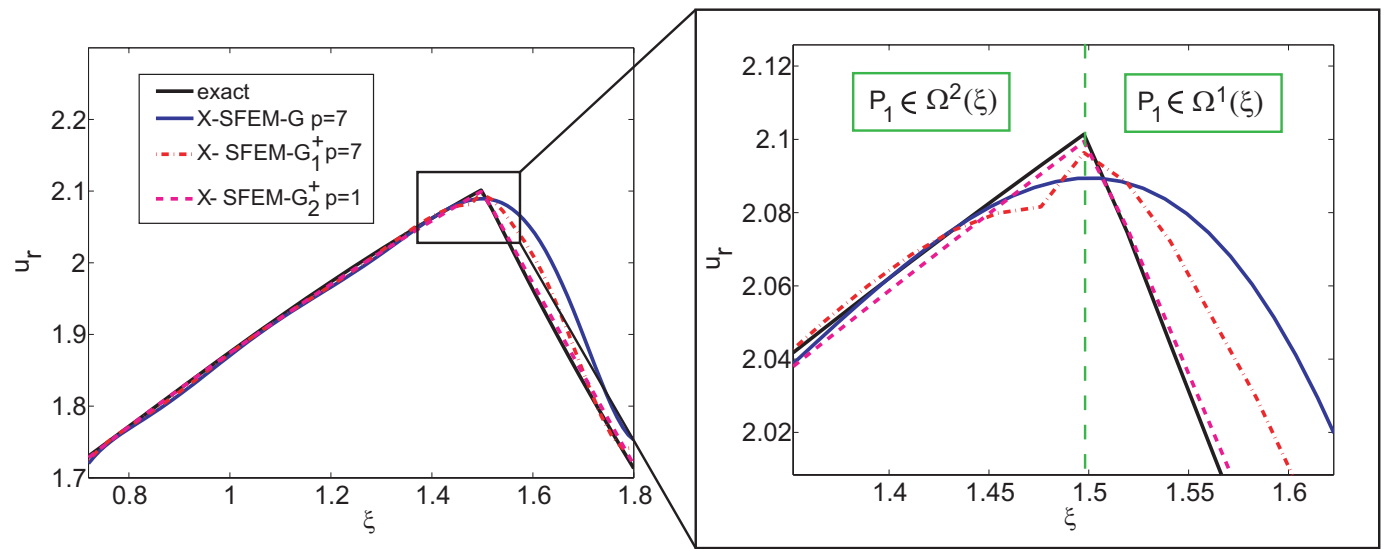

Figure 15. Example 2: response surfaces of radial displacement for point $P_{1}$ obtained with X-SFEM-G $(p=7), \mathrm{X}-\mathrm{SFEM}-\mathrm{G}_{1}^{+}(p=7)$ and X-SFEM-G ${ }_{2}^{+}(p=1)$.

Random variables $\xi_{1} \in U(-1,1)$ and $\xi_{2} \in U(-1,1)$ are statistically independent uniform random variables. We work in the associated 2-dimensional probability space $\left(\boldsymbol{\Xi}, \mathcal{B}_{\boldsymbol{\Xi}}, P_{\boldsymbol{\xi}}\right)$ with $\boldsymbol{\xi}=\left(\xi_{1}, \xi_{2}\right)$ and $\boldsymbol{\Xi}=(-1,1) \times(-1,1)$. The material parameters of the two phases are deterministic: $E_{1}=1$ and $\nu_{1}=0.3$ in $\Omega^{1}(\boldsymbol{\xi})$ and $E_{2}=10$ and $\nu_{2}=0.3$ in $\Omega^{2}(\boldsymbol{\xi})$.

In this example, we want to compute the random homogenized elasticity tensor $\boldsymbol{C}^{\text {hom }}(\boldsymbol{\xi})$ leading to a macroscopic constitutive relation $\boldsymbol{\Sigma}=\boldsymbol{C}^{\text {hom }(\boldsymbol{\xi})}: \boldsymbol{E}$, linking the macroscopic stress field $\boldsymbol{\Sigma}$ to the macroscopic strain field $\boldsymbol{E}$. The chosen homogenization procedure consists in imposing homogeneous stresses on the boundary [42], i.e. $\boldsymbol{F}=\boldsymbol{\Sigma} \cdot \boldsymbol{n}$ on $\partial \Omega$. In practise, we compute the solutions corresponding to the three loading cases shown on Figure 16 (uniform horizontal tension, uniform vertical tension and homogeneous shear), corresponding to the three following macroscopic stresses:

$$
\boldsymbol{\Sigma}^{(11)}=\left(\begin{array}{cc}
1 & 0 \\
0 & 0
\end{array}\right), \quad \boldsymbol{\Sigma}^{(22)}=\left(\begin{array}{cc}
0 & 0 \\
0 & 1
\end{array}\right), \quad \boldsymbol{\Sigma}^{(12)}=\left(\begin{array}{cc}
0 & 1 \\
1 & 0
\end{array}\right)
$$

We denote by $\boldsymbol{u}^{(k l)}(\boldsymbol{x}, \boldsymbol{\xi})$ the three corresponding displacement fields. The coefficients of the random homogenized compliance $\boldsymbol{S}^{\mathrm{hom}}(\boldsymbol{\xi})=\boldsymbol{C}^{\mathrm{hom}}(\boldsymbol{\xi})^{-1}$ are then obtained by the following equations:

$$
\left(\boldsymbol{S}^{\mathrm{hom}}(\boldsymbol{\xi})\right)_{i j k l}=\frac{1}{m e s(\Omega)} \int_{\partial \Omega}\left(\boldsymbol{u}^{(k l)} \otimes^{s} \boldsymbol{n}\right)_{i j} d s
$$

At the spatial level, we use the finite element mesh shown on Figure 17. At stochastic level, we use a generalized polynomial chaos with degree $p=3$ at stochastic level. In this example, we only consider the approximate solutions obtained with X-SFEM-G and X-SFEM-G ${ }_{2}^{+}$(Galerkin projections).

7.4.2. Comparison with a deterministic X-FEM approach Here, we compare the approximate solutions obtained with X-SFEM-G and X-SFEM-G $\mathrm{G}_{2}^{+}$with a reference solution obtained with a simple sampling technique coupled with a deterministic X-FEM code (with a classical 

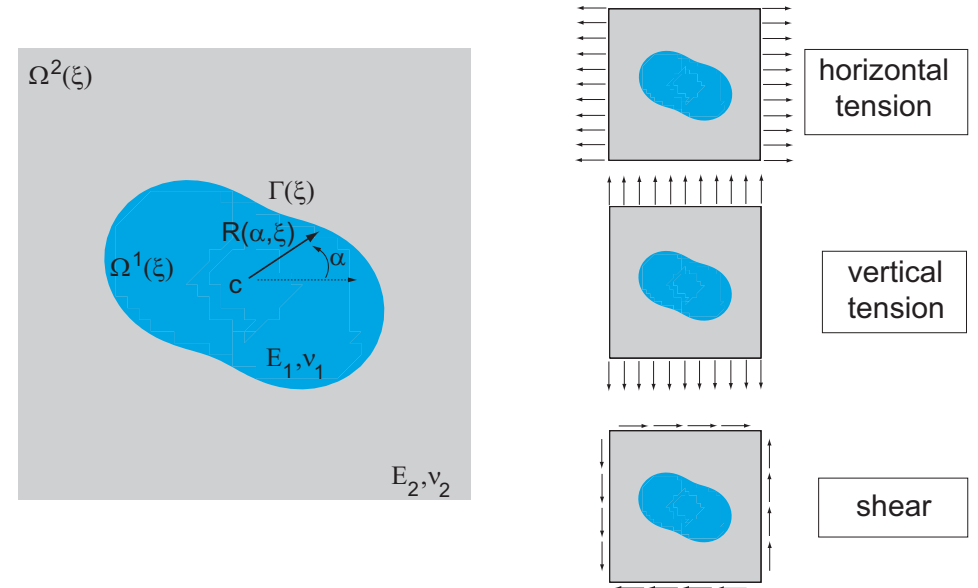

Figure 16. Example 3: cell of an heterogenous material with random inclusion.

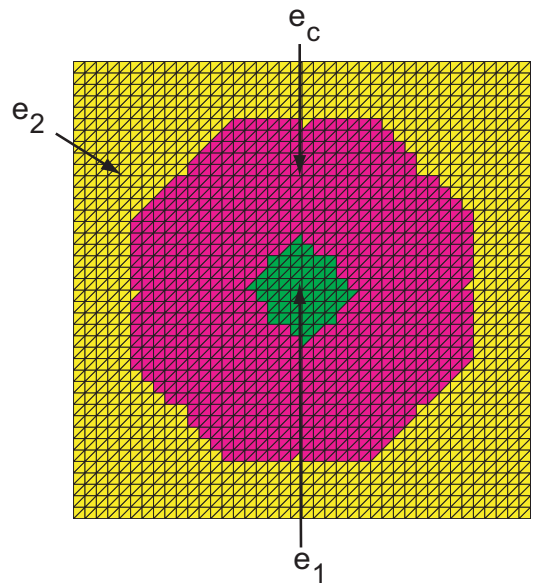

Figure 17. Example 3: X-SFEM finite element mesh with the three element groups: $\left(e_{1}\right)$ gathers elements surely in $\Omega^{1},\left(e_{2}\right)$ gathers elements surely in $\Omega^{2}$ and $\left(e_{c}\right)$ gathers elements possibly cut by the interface $\Gamma(\boldsymbol{\xi})$.

enrichment based on $\left.\left.\psi_{1}\right)\right)$. We choose a particular point $\boldsymbol{x}=(0.7,0.7)$ of $\Omega$ such that $P_{\boldsymbol{\xi}}\left(\boldsymbol{x} \in \Omega^{k}(\boldsymbol{\xi})\right)>0$ for $k=1$ and 2 . We first look at the response surfaces of the horizontal displacement at $\boldsymbol{x}$ for the case of homogeneous shear loading. Figure 18 shows the response surfaces obtained with X-SFEM-G, X-SFEM-G ${ }_{2}^{+}$and the reference deterministic X-FEM solution. We observe that the $\mathrm{X}-\mathrm{SFEM}-\mathrm{G}_{2}^{+}$solution is very close to the reference solution while the X-SFEM-G solution gives quite bad results. It is to note that the enriched approximation based on function $\psi_{2}$ is able to capture the strong irregularity in the response surface and allows the use of a simple polynomial chaos basis for the representation of degrees of freedom. Figure 19 shows the stress component $\sigma_{11}$ obtained for two particular outcomes of the geometry 
and for two different loading cases. We notice that stresses obtained with the X-SFEM-G ${ }_{2}^{+}$ solution match very well the reference solution near the interface, which is not the case of the standard X-SFEM-G approximation.

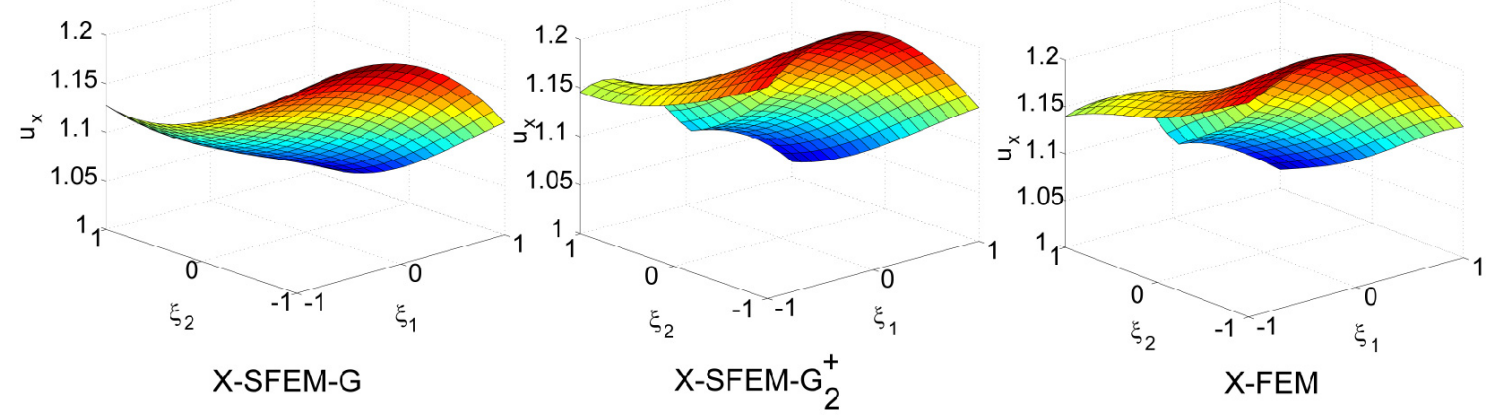

Figure 18. Example 3: response surfaces of horinzontal displacement for point $\boldsymbol{x}=(0.7,0.7)$ obtained with X-SFEM-G, X-SFEM-G ${ }_{2}^{+}$and the reference solution (X-FEM); shear loading case.

7.4.3. Computation of homogenized stiffness Now, we focus on the calculation of the random homogenized elasticity tensor $\boldsymbol{C}^{\text {hom }}(\boldsymbol{\xi})$. The different displacement fields $\boldsymbol{u}^{(k l)}$ are obtained with X-SFEM-G or X-SFEM-G ${ }_{2}^{+}$projections. Then, in a post-processing step, we compute a decomposition of $\boldsymbol{C}^{\text {hom }}(\boldsymbol{\xi})$ on a polynomial chaos basis of degree $p=3: \boldsymbol{C}^{\text {hom }}(\boldsymbol{\xi})=$ $\sum_{\alpha=1}^{P} C_{\alpha}^{\text {hom }} H_{\alpha}(\boldsymbol{\xi})$. The reference response surface is obtained with deterministic X-FEM computations, with a classical enrichment based on function $\psi_{1}$. Figure 20 shows the response surfaces of $C_{1111}^{\text {hom }}$ obtained with X-SFEM-G, X-SFEM-G ${ }_{2}^{+}$and the reference solution. We clearly observe that X-SFEM-G ${ }_{2}^{+}$leads to far better results than X-SFEM-G.

Figures 21(a) and 21(b) illustrate the probability density functions (PDFs) obtained for two components of $C^{\text {hom }}$. As a reference solution, we use a Monte-Carlo approach with 5,000 samplings coupled to a deterministic X-FEM code with enrichment based on function $\psi_{1}$. We can notice a good agreement between $\mathrm{X}-\mathrm{SFEM}-\mathrm{G}_{2}^{+}$and the reference solution.

Finally, we focus on the two first statistical moments of the components of $\boldsymbol{C}^{\text {hom }}$. Table III gives the mean $\mu$ and the standard deviation $\sigma$ of $C_{1111}^{\text {hom }}$ and $C_{1122}^{\text {hom }}$ obtained with X-SFEM-G, $\mathrm{X}-\mathrm{SFEM}-\mathrm{G}_{2}^{+}$and a reference Monte-Carlo method coupled with a deterministic X-FEM code. We observe a very good agreement between the X-SFEM-G $\mathrm{G}_{2}^{+}$and the reference Monte-Carlo simulation. The bad results obtained with X-SFEM-G method clearly highlight the relevance of the enrichment procedure.

\subsection{Example 4: heterogeneous cell with material interface presenting changes in topology}

This example will illustrate the ability of the X-SFEM technique to deal with changes of topology in the geometry. We consider a cell $\Omega=(0,1) \times(0,1)$ of an heterogeneous material composed of two material phases. The material parameters of the two phases are deterministic: $E_{1}=10$ and $\nu_{1}=0.3$ in $\Omega^{1}$ and $E_{2}=1$ and $\nu_{2}=0.25$ in $\Omega^{2}$. The random domain $\Omega^{2}(\boldsymbol{\xi})$ is the union of two circular inclusions with radius $r=0.15$ and random horizontal location (see Figure 22(a)). The two phases $\Omega^{1}(\boldsymbol{\xi})$ and $\Omega^{2}(\boldsymbol{\xi})$ are separated by a random interface $\Gamma(\boldsymbol{\xi})$ 


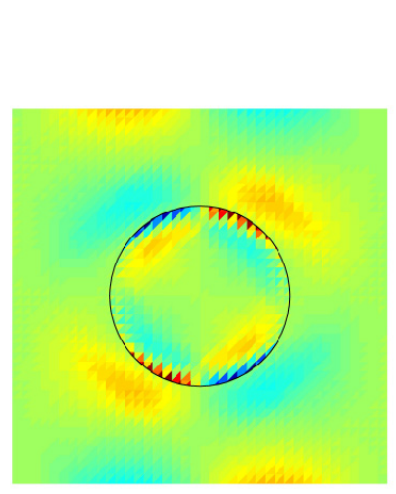

(a)

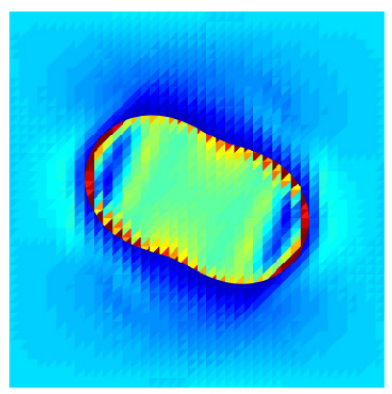

(a)

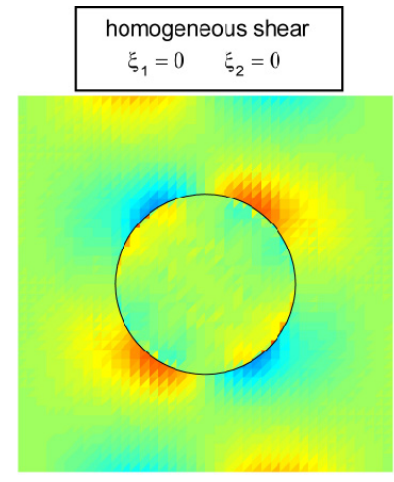

(b)

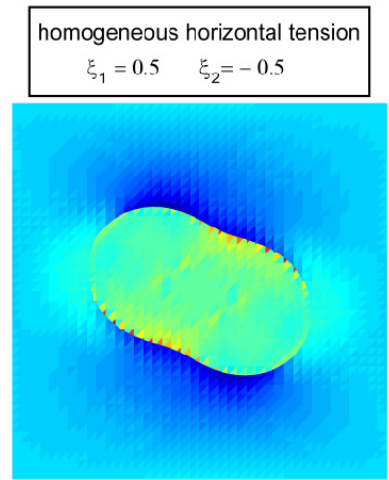

(b)

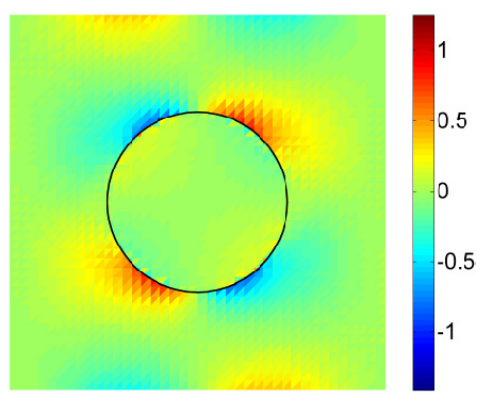

(c)

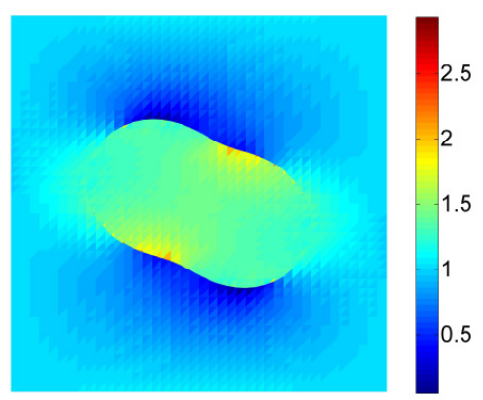

(c)

Figure 19. Exemple 3: comparison of stress fields $\sigma_{11}$ obtained with X-SFEM-G (a), X-SFEM-G ${ }_{2}^{+}$(b) and reference X-FEM (c) for two outcomes of the geometry and two loading cases.

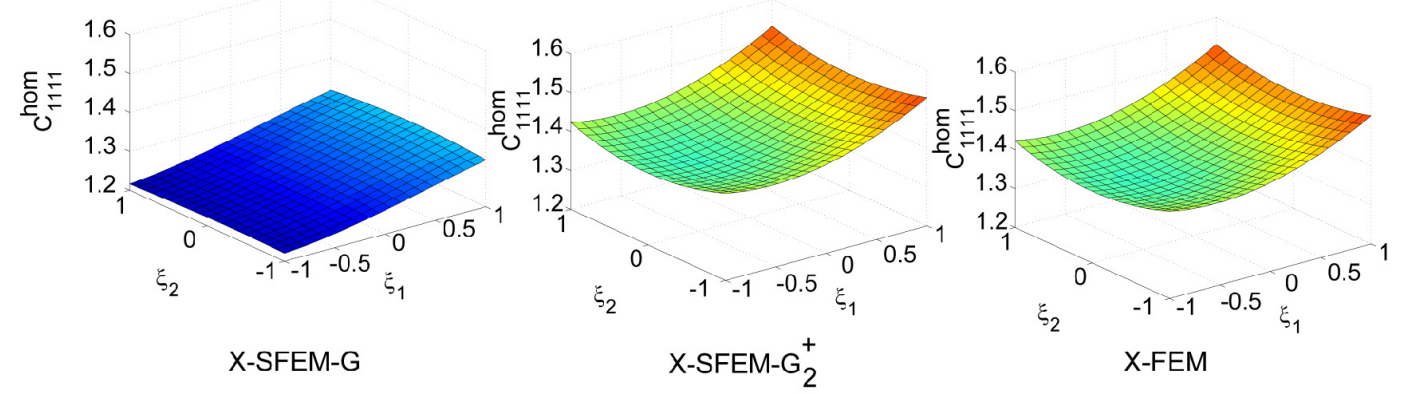

Figure 20. Exemple 3: response surfaces of $\boldsymbol{C}_{1111}^{\text {hom }}$ obtained with X-SFEM-G, X-SFEM-G ${ }^{+} 2$ and the reference solution (X-FEM). 


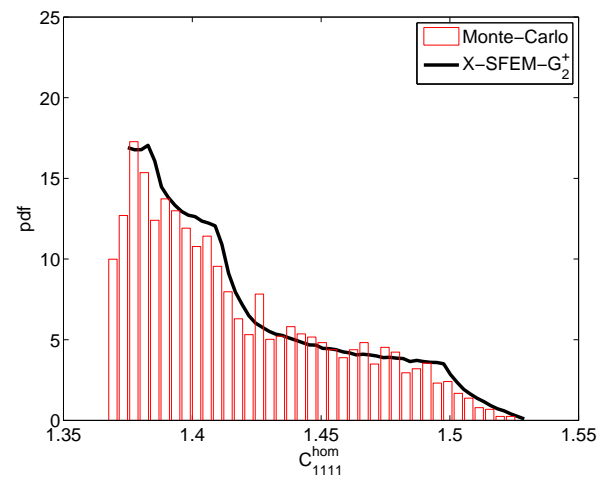

(a)

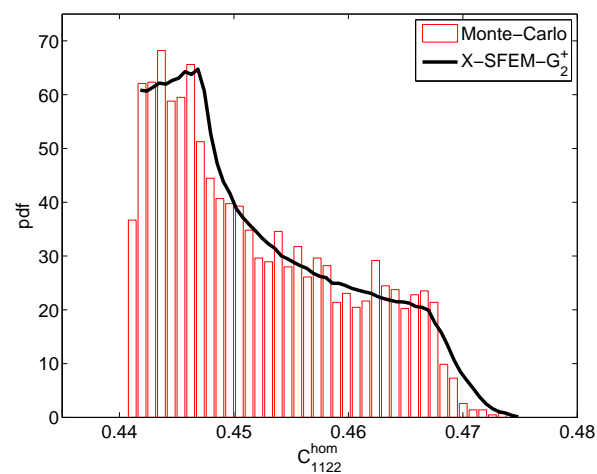

(b)

Figure 21. Example 3: probability density functions of $\boldsymbol{C}_{1111}^{\text {hom }}$ and $\boldsymbol{C}_{1122}^{\text {hom }}$ obtained with X-SFEM-G ${ }_{2}^{+}$ and the reference solution (Monte-Carlo with 5,000 samples).

\begin{tabular}{|c|c|c|c|c|}
\hline & \multicolumn{2}{|c|}{$C_{1111}^{\text {hom }}$} & \multicolumn{2}{|c|}{$C_{1122}^{\text {hom }}$} \\
\hline & $\mu$ & $\sigma$ & $\mu$ & $\sigma$ \\
\hline X-SFEM-G & 1.268 & 0.0323 & 0.4118 & 0.0043 \\
\hline X-SFEM-G ${ }_{2}^{+}$ & 1.4201 & 0.0379 & 0.4523 & 0.008 \\
\hline Monte-Carlo/X-FEM & $\begin{array}{c}1.4165 \\
\pm 4.10^{-4}\end{array}$ & $\begin{array}{c}0.0376 \\
\pm 1.10^{-4}\end{array}$ & $\begin{array}{c}0.4519 \\
\pm 4.10^{-5}\end{array}$ & $\begin{array}{c}0.0081 \\
\pm 2.10^{-5}\end{array}$ \\
\hline
\end{tabular}

Table III. Example 3: mean $\mu$ and standard deviation $\sigma$ of $C_{1111}^{\text {hom }}$ and $C_{1122}^{\text {hom }}$ obtained with X-SFEM-G, $\mathrm{X}-\mathrm{SFEM}-\mathrm{G}_{2}^{+}$and the reference Monte-Carlo/X-FEM method (with confidence intervals).

characterized by the iso-zero of the following level-set:

$$
\phi(\boldsymbol{x}, \boldsymbol{\xi})=\max \left(\phi_{1}(\boldsymbol{x}, \boldsymbol{\xi}), \phi_{2}(\boldsymbol{x}, \boldsymbol{\xi})\right), \quad \phi_{i}=r-\left\|\boldsymbol{x}-\left(\xi_{i}, 0.5\right)\right\|
$$

where $\xi_{1} \in U(0.2,0.5)$ and $\xi_{2} \in U(0.5,0.8)$ are statistically independent uniform random variables. We work in the associated 2-dimensional probability space $\left(\boldsymbol{\Xi}, \mathcal{B}_{\boldsymbol{\Xi}}, P_{\boldsymbol{\xi}}\right)$ with $\boldsymbol{\xi}=$ $\left(\xi_{1}, \xi_{2}\right)$ and $\boldsymbol{\Xi}=(0.2,0.5) \times(0.5,0.8)$.

In this example, we only consider the approximation obtained with X-SFEM-G ${ }_{2}^{+}$(Galerkin projection on an approximation space enriched with function $\psi_{2}$ ). At the stochastic level, we use a polynomial chaos of degree $p=3$ in dimension 2 . The finite element mesh is shown on figure $22(\mathrm{~b})$. Let us note that there is no element surely in the domain $\Omega^{2}$. Figure $22(\mathrm{c})$ shows the set of enriched finite elements $\mathcal{T}_{h}^{+}$.

Figure 23 shows the stress component $\sigma_{11}$ obtained for four particular outcomes of the geometry (with different topologies). For all outcomes, we notice that stresses obtained with the X-SFEM-G $\mathrm{X}_{2}^{+}$solution match very well the reference solution, computed with a classical deterministic X-FEM method (with $\psi_{1}$ enrichment). The proposed X-SFEM approach allows to build an approximate solution which is very accurate for all outcomes of the geometry although these outcomes correspond to very different geometrical patterns, with eventual changes in topology. 


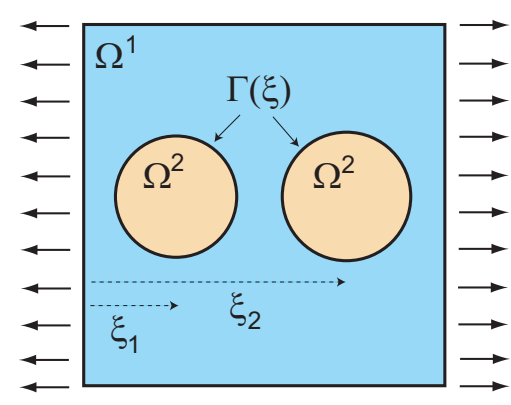

(a)

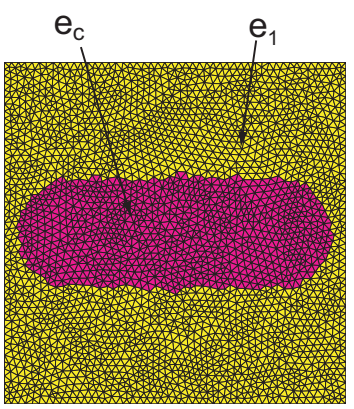

(b)

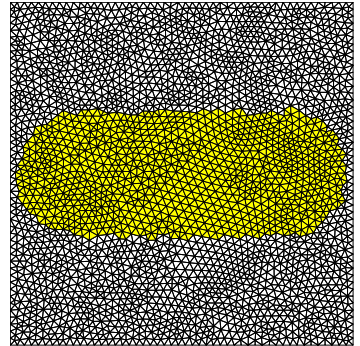

(c)

Figure 22. Example 4: (a) problem definition. (b) finite element mesh with the two element groups: elements $e_{1}$ surely in $\Omega^{1}$, elements $e_{c}$ possibly cut by the interface $\Gamma(\boldsymbol{\xi})$ (no elements surely in $\Omega^{2}$ ).

(c) Enriched finite elements $K \in \mathcal{T}_{h}^{+}$.

$\xi_{1}$

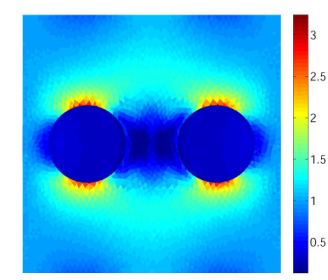

(b)

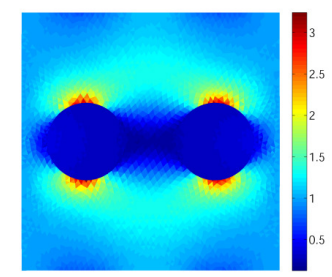

$\xi_{2}$
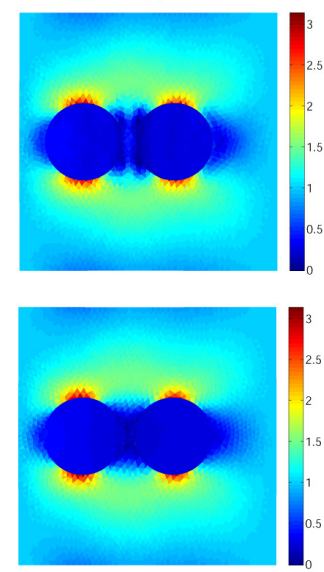

$\xi_{3}$
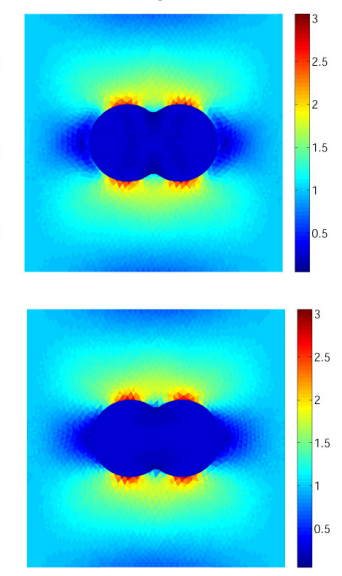

$\xi_{4}$
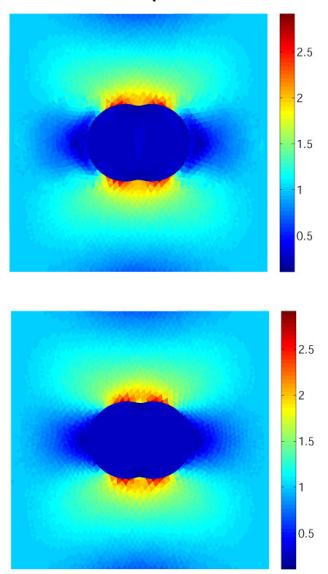

Figure 23. Exemple 4: comparison of stress fields $\sigma_{11}$ obtained with X-SFEM-G ${ }_{2}^{+}$(a) and reference $\mathrm{X}-\mathrm{FEM}$ (b) for four outcomes of the geometry (corresponding to two types of topologies).

We now consider the computation of the elastic energy $W(\boldsymbol{\xi})$ defined by

$$
W(\boldsymbol{\xi})=\frac{1}{2} \int_{\Omega} \boldsymbol{\epsilon}(\boldsymbol{u}(\boldsymbol{x}, \boldsymbol{\xi})): \boldsymbol{C}: \boldsymbol{\epsilon}(\boldsymbol{u}(\boldsymbol{x}, \boldsymbol{\xi})) d \boldsymbol{x}
$$

Figure 24 illustrates the probability density functions of $W(\boldsymbol{\xi})$ obtained with the X-SFEM$\mathrm{G}_{2}^{+}$solution and a reference solution based on a Monte-Carlo approach with 10,000 samples coupled with a deterministic X-FEM method using a classical $\psi_{1}$ enrichment. We can observe a good agreement between the two solutions.

Finally, Table IV indicates the tail probabilities of $W(\boldsymbol{\xi})$ obtained with $\mathrm{X}-\mathrm{SFEM}-\mathrm{G}_{2}^{+}$for different orders $p \in\{3,4,5\}$. Results are compared with the reference Monte-Carlo simulation. We observe a very good accuracy of X-SFEM-G $\mathrm{G}_{2}^{+}$for the estimation of events with probabilities 


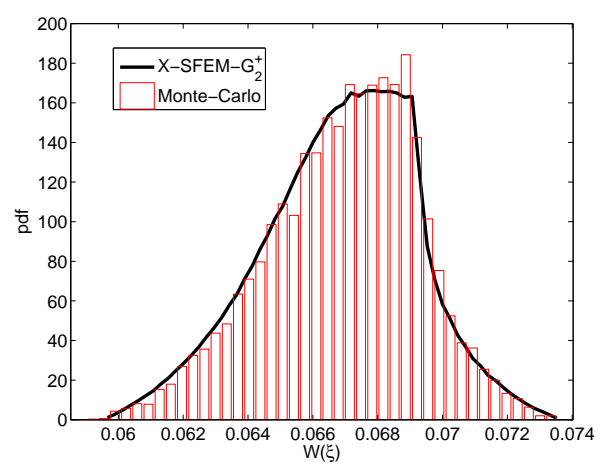

Figure 24. Exemple 4: comparison of probability density functions of $W(\boldsymbol{\xi})$ obtained with X-SFEM-G $\mathrm{G}_{2}^{+}$ and the reference solution (Monte-Carlo with 10,000 samples).

down to $10^{-3}$, even with low polynomial degree $p$. Let us note that in order to obtain such a good accuracy for lower probabilities, a higher polynomial degree $p$ could be used.

\begin{tabular}{|c|c|c|c|c|c|}
\cline { 3 - 6 } \multicolumn{2}{c|}{} & \multicolumn{4}{|c|}{$w$} \\
\cline { 3 - 6 } \multicolumn{2}{c|}{} & 0.07 & 0.071 & 0.072 & 0.073 \\
\hline \multirow{3}{*}{ X-SFEM-G $_{2}^{+}$} & $p=3$ & $7.510^{-2}$ & $3.210^{-2}$ & $1.0510^{-2}$ & $1.410^{-3}$ \\
\cline { 2 - 6 } & $p=4$ & $7.510^{-2}$ & $3.210^{-2}$ & $1.0210^{-2}$ & $1.110^{-3}$ \\
\cline { 2 - 6 } & $p=5$ & $7.510^{-2}$ & $3.210^{-2}$ & $0.9910^{-2}$ & $1.110^{-3}$ \\
\hline \multicolumn{2}{|c|}{ Monte-Carlo/X-FEM } & $7.610^{-2}$ & $3.110^{-2}$ & $0.9510^{-3}$ & $0.910^{-3}$ \\
& $\pm 1.10^{-3}$ & $\pm 7.10^{-4}$ & $\pm 4.10^{-4}$ & $\pm 1.10^{-4}$ \\
\hline
\end{tabular}

Table IV. Example 4: probabilities $P_{\boldsymbol{\xi}}(W(\boldsymbol{\xi})>w)$ obtained with X-SFEM-G $\mathrm{G}_{2}^{+}$and a Monte-Carlo/XFEM method (with 10,000 samples), for different values of $w$.

\section{CONCLUSIONS, LIMITATIONS AND PERSPECTIVES}

We have proposed a mariage between the eXtended Finite Element Method (X-FEM) and spectral stochastic methods for the numerical simulation of multi-phased heterogeneous materials with random material interfaces. The first point of the proposed method consists in representing implicitly the random geometry with the level-set technique. Classical spectral stochastic finite element approximation spaces are then simply defined by introducing a fixed finite element mesh. However, these classical approximation spaces (finite element at space level and polynomial chaos at stochastic level) do not allow capturing the irregularities of the solution field with respect to spatial and stochastic variables. Then, an extension of the partition of unity method has been introduced for the construction of enriched approximation spaces in a tensor product space, which are able to capture these irregularities and lead to better accuracy and convergence properties of the approximation. Two enrichment strategies (choices 1 and 2) have been proposed and compared. The choice 1, directly inspired from the deterministic X-FEM, appeared to be unadapted to the stochastic framework. The choice 2, 
which is adapted to the spectral stochastic framework, allows to recover very good convergence properties of the approximation at both spatial and stochastic levels. It has been also illustrated that both Galerkin and $L^{2}$ stochastic projections lead to satisfactory results when using the proposed enriched approximation spaces.

A natural extension of this work concerns the study of structures containing cracks with random location or geometry. This extension will be introduced in a forthcoming paper.

Let us emphasize that the proposed method allows handling problems where the randomness on the geometry of interfaces can be represented by a low number of random parameters. This limitation is classical in the context of spectral stochastic methods and is due to the dramatic increase of computational costs when dealing with high stochastic dimension (so called curse of dimensionality). This clearly restricts the range of application of the proposed method. In particular, in its present form, this method can not be applied for the homogenization of multiphased random materials, where the description of the random microstructure (or equivalently of the random interface between phases) can typically require the introduction of thousands of random variables $[43,44]$. Until now, traditional stochastic simulation techniques, associated with deterministic FEM or XFEM-type approaches, remain the only way to perform such computational analyses. However, methodologies based on separated representation techniques have been recently proposed in order to circumvent the curse of dimensionality in the context of spectral approaches [45]. The coupling between these techniques and the developments of the present paper will be addressed in future works. Let us note that alternative to spectral stochastic methods are available for constructing an explicit representation of a function of random parameters. In particular, for high stochastic dimension, different methods have been proposed in order to circumvent the curse of dimensionality: ANOVA, HDMR, polynomial dimensional decomposition [46, 47, 48]. For the representation of functionals which do not satisfy the basic assumptions required by these techniques (e.g. low order correlations amongst input variables), enrichment strategies inspired from the present article may be a possible remedy.

Another point concern the extension of the proposed method to the case of evolving random interfaces. For time-dependent stochastic problems, the accuracy of spectral approaches based on polynomial approximations may strongly deteriorate. Let us finally mention that the extension of the proposed enrichment strategy to time-dependent problems could be a possible way to circumvent this drawback.

\section{REFERENCES}

1. Ghanem R. Ingredients for a general purpose stochastic finite elements implementation. Computer Methods in Applied Mechanics and Engineering 1999; 168:19-34.

2. Xiu D. Fast numerical methods for stochastic computations: a review. Comm. Comput. Phys. 2009; 5:242272 .

3. Nouy A. Recent developments in spectral stochastic methods for the numerical solution of stochastic partial differential equations. Archives of Computational Methods in Engineering 2009; 16(3):251-285.

4. Matthies HG. Stochastic finite elements: Computational approaches to stochastic partial differential equations. Zamm-Zeitschrift Fur Angewandte Mathematik Und Mechanik 2008; 88(11):849-873.

5. Babuška I, Tempone R, Zouraris GE. Solving elliptic boundary value problems with uncertain coefficients by the finite element method: the stochastic formulation. Computer Methods in Applied Mechanics and Engineering 2005; 194:1251-1294.

6. Matthies HG, Keese A. Galerkin methods for linear and nonlinear elliptic stochastic partial differential equations. Computer Methods in Applied Mechanics and Engineering 2005; 194(12-16):1295-1331. 
7. Frauenfelder P, Schwab C, Todor RA. Finite elements for elliptic problems with stochastic coefficients. Computer Methods in Applied Mechanics and Engineering 2005; 194(2-5):205-228.

8. Babuška I, Nobile F, Tempone R. A stochastic collocation method for elliptic partial differential equations with random input data. SIAM J. Num. Anal. 2007; 45(3):1005-1034.

9. F Nobile CW R Tempone. A sparse grid stochastic collocation method for partial differential equations with random input data. SIAM Journal on Numerical Analysis 2007; 46(5):2309-2345.

10. Xiu D, Hesthaven J. High-order collocation methods for differential equations with random inputs. SIAM J. Sci. Comput. 2005; 27(3):1118Ü1139.

11. Xiu D, Ganapathysubramanian B, Zabaras N. Sparse grid collocation schemes for stochastic natural convection problems. Journal of Computational Physics 2007; 225(1):652-685.

12. Xiu D. Efficient collocational approach for parametric uncertainty analysis. Comm. Comput. Phys. 2007; 2(2):293-309.

13. Ghiocel D, Ghanem R. Stochastic finite-element analysis of seismic soil-structure interaction. $A S C E$ Journal Engrg. Mech. 2002; 128(1):66-77.

14. Keese A. A review of recent developments in the numerical solution of stochastic pdes (stochastic finite elements). Technical Report 2003-6, Technical report, Institute of Scientific Computing, Tech. Univ. Braunschweig, Germany 2003. Http://opus.tu-bs.de/opus/volltexte/2003/504/.

15. Reagan M, Najm H, Ghanem R, Knio O. Uncertainty quantification in reacting flow simulations through non-intrusive spectral projection. Combustion and Flames 2003; 132:545-555.

16. Blatman G, Sudret B. Sparse polynomial chaos expansions and adaptive stochastic finite elements using a regression approach. Comptes Rendus Mécanique 2007; 336(6):518-523.

17. Niederreiter H. Random Number Generation and quasi-Monte Carlo Methods. SIAM, Philadelphia, PA, 1992.

18. Tartakovsky DM, Xiu D. Stochastic analysis of transport in tubes with rough walls. Journal of Computational Physics 2006; 217:248-259.

19. Canuto C, Kozubek T. A fictitious domain approach to the numerical solution of pdes in stochastic domains. Numerische Mathematik 2007; 107(2):257-293.

20. Nouy A, Schoefs F, Moës N. X-SFEM, a computational technique based on X-FEM to deal with random shapes. European Journal of Computational Mechanics 2007; 16(2):277-293.

21. Nouy A, Clément A, Schoefs F, Moës N. An extended stochastic finite element method for solving stochastic partial differential equations on random domains. Computer Methods in Applied Mechanics and Engineering 2008; 197:4663-4682.

22. Moës N, Dolbow J, Belytschko T. A finite element method for crack growth without remeshing. Int. J. for Numerical Methods in Engineering 1999; 46:131-150.

23. Sukumar N, Chopp D, Moës N, Belytschko T. Modeling holes and inclusions by level sets in the extended finite-element method. Computer Methods in Applied Mechanics and Engineering 2001; 190:6183-6200.

24. Melenk JM, Babuška I. The partition of unity method: basic theory and applications. Computer Methods in Applied Mechanics and Engineering 1996; 39:289-314.

25. Ghosh D, Ghanem R. Stochastic convergence acceleration through basis enrichment of polynomial chaos expansions. Int. J. for Numerical Methods in Engineering 2008; 73:162-184.

26. Sethian J. Level Set Methods and Fast Marching Methods: Evolving Interfaces in Computational Geometry, Fluid Mechanics, Computer Vision, and Materials Science. Cambridge University Press, Cambridge, UK, 1999.

27. Stefanou G, Nouy A, Clément A. Identification of random shapes from images through polynomial chaos expansion of random level-set functions. Int. J. for Numerical Methods in Engineering 2009; 79(2):127155.

28. Ghanem R, Spanos P. Stochastic finite elements: a spectral approach. Springer, Berlin, 1991.

29. Xiu D, Karniadakis GE. The Wiener-Askey polynomial chaos for stochastic differential equations. SIAM J. Sci. Comput. 2002; 24(2):619-644.

30. Deb M, Babuška I, Oden JT. Solution of stochastic partial differential equations using galerkin finite element techniques. Computer Methods in Applied Mechanics and Engineering 2001; 190:6359-6372.

31. Le Maître O, Knio OM, Najm HN, Ghanem RG. Uncertainty propagation using Wiener-Haar expansions. Journal of Computational Physics 2004; 197(1):28-57.

32. Wan X, Karniadakis G. An adaptive multi-element generalized polynomial chaos method for stochastic diffential equations. J. Comp. Phys. 2005; 209:617-642.

33. Soize C, Ghanem R. Physical systems with random uncertainties: chaos representations with arbitrary probability measure. SIAM J. Sci. Comput. 2004; 26(2):395-410.

34. Keese A, Mathhies HG. Hierarchical parallelisation for the solution of stochastic finite element equations. Computer Methods in Applied Mechanics and Engineering 2005; 83:1033-1047.

35. Nouy A. A generalized spectral decomposition technique to solve a class of linear stochastic partial differential equations. Computer Methods in Applied Mechanics and Engineering 2007; 196(45-48):4521- 
4537.

36. Nouy A. Generalized spectral decomposition method for solving stochastic finite element equations: invariant subspace problem and dedicated algorithms. Computer Methods in Applied Mechanics and Engineering 2008; 197:4718-4736.

37. Nouy A, Le Maître O. Generalized spectral decomposition method for stochastic non linear problems. Journal of Computational Physics 2009; 228(1):202-235.

38. Moës N, Cloirec M, Cartraud P, Remacle J. A computational approach to handle complex microstructure geometries. Comp. Meth. App. Mech. Eng. 2003; 192:3163-3177.

39. Dolbow J, Moës N, Belytschko T. Discontinuous enrichment in finite elements with a partition of unity method. Finite Elements in Analysis and Design 2000; 36(3-4):235-260.

40. Strouboulis T, Babuška I, Copps K. The design and analysis of the Generalized Finite Element Method. Computer Methods in Applied Mechanics and Engineering 2000; 182:43-71.

41. Le Maître O, Najm H, Ghanem R, Knio O. Multi-resolution analysis of Wiener-type uncertainty propagation schemes. Journal of Computational Physics 2004; 197(2):502-531.

42. Zohdi TI. Homogenization methods and multiscale modeling. Encyclopedia of Computational Mechanics, vol. 2, chapter 12, Wiley, New York, 2004.

43. Chakraborty A, Rahman S. Stochastic multiscale models for fracture analysis of functionally graded materials. Engineering Fracture Mechanics 2008; 75(8):2062-2086.

44. Chakraborty A, Rahman S. A parametric study on probabilistic fracture of functionally graded composites by a concurrent multiscale method. Probabilistic Engineering Mechanics 2009; 24(3):438-451.

45. Nouy A. Proper generalized decompositions and separated representations for the numerical solution of high dimensional stochastic problems. Archives of Computational Methods in Engineering, In Press.

46. Efron B, Stein C. The jackknife estimate of variance. Ann. Statist. 1981; 9(3):586-596.

47. Rabitz H, Alis O. General foundations of high-dimensional model representations. Journal Of Mathematical Chemistry 1999; 25(2-3):197-233.

48. Rahman S. A polynomial dimensional decomposition for stochastic computing. International Journal For Numerical Methods In Engineering 2008; 76(13):2091-2116. 